Article

\title{
Scanning Photogrammetry for Measuring Large Targets in Close Range
}

\author{
Shan Huang, Zuxun Zhang, Tao Ke *, Min Tang and Xuan Xu
}

School of Remote Sensing and Information Engineering, Wuhan University, No.129 Luoyu Road, Wuhan 430079, China; E-Mails: huangsh@whu.edu.cn (S.H.); zhangzx@cae.cn (Z.Z.); min.tang@whu.edu.cn (M.T.); xxuwhu@sina.com (X.X.)

* Author to whom correspondence should be addressed; E-Mail: ketao@whu.edu.cn; Tel.: +86-135-5443-5928; Fax: +86-27-6877-8010.

Academic Editors: Diego Gonzalez-Aguilera, Fabio Remondino, Henrique Lorenzo and Prasad S. Thenkabail

Received: 5 May 2015 / Accepted: 29 July 2015 / Published: 7 August 2015

\begin{abstract}
In close-range photogrammetry, images are difficult to acquire and organize primarily because of the limited field of view (FOV) of digital cameras when long focal lenses are used to measure large targets. To overcome this problem, we apply a scanning photography method that acquires images by rotating the camera in both horizontal and vertical directions at one station. This approach not only enlarges the FOV of each station but also ensures that all stations are distributed in order without coverage gap. We also conduct a modified triangulation according to the traits of the data overlapping among images from the same station to avoid matching all images with one another. This algorithm synthesizes the images acquired from the same station into synthetic images, which are then used to generate a free network. Consequently, we solve the exterior orientation elements of each original camera image in the free network and perform image matching among original images to obtain tie points. Finally, all original images are combined in self-calibration bundle adjustment with control points. The feasibility and precision of the proposed method are validated by testing it on two fields using 300 and $600 \mathrm{~mm}$ lenses. The results confirm that even with a small amount of control points, the developed scanning photogrammetry can steadily achieve millimeter scale accuracy at distances ranging from $40 \mathrm{~m}$ to $250 \mathrm{~m}$.
\end{abstract}

Keywords: scanning photography; close-range photogrammetry; modified triangulation; long focal lens 


\section{Introduction}

The development of electric sensors and image processing techniques has contributed to the rapid growth of the application of photogrammetry. Since 2000, photogrammetric mapping using digital photographic systems has become popular because of the application of digital cameras [1-6]. Given their changeable focus lens, various photography methods, and low cost, digital single-lens reflex cameras (DSLRs) are widely applied in close-range measurement tasks [7-13]. The high resolution of DSLRs is helpful for high-precision measurements. These advantages suggest the need to develop photogrammetry systems that can help inexperienced users accomplish close-range measurements well $[11,12,14]$. The size of a single digital image sensor (e.g., charge-coupled device (CCD) detector) is limited. As such, the field of view (FOV) of a digital image captured by this sensor is restricted compared with that of film image with the same focal length. Therefore, when digital cameras with long focal lens are used for measurements, the limited intersection angle decreases the measurement precision if a normal photography method is employed. Oblique photography can ensure intersection angles and maintain high measurement accuracy [8,15-18]. However, when the intersection angle increases, the parallax of correspondences changes in a large range and may become discontinuous as the occlusion occurs. Large intersection angles make image matching difficult, whereas small ones result in low intersection precision. Therefore, in many measurement methods, multi-view photography is applied to solve this contradiction [19-21]. Nonetheless, this technique introduces new problems, such as the difficult acquisition of images in order, particularly in large measurement ranges. Likewise, organizing images for data processing is complicated. There is a lot of work to be done to solve these issues.

In aerial photogrammetry, several approaches for expanding the format of cameras have been proposed (e.g., UltraCam and digital mapping cameras) to facilitate the continuous acquisition of nadir images. These aerial cameras consist of a specific number of fixed CCD detectors and employ a special software to make the frames completely stitched into a whole image. The stitched images are then used in traditional triangulation method. However, the portability of these aerial cameras is crucial to fulfilling close-range measurements tasks. Therefore, multi-view photography by one camera is a widely adopted alternative technique to "normal" photography to ensure intersection angles and maintain high measurement accuracy [8,15-18]. In this case, the approach of matching all images to one another and certain improvements of the method are proposed to determine the relationship among images for automatic image organization. To ensure measurement accuracy, numerous ground control points are employed as in V-STARS [22] and ACION [23]. Nevertheless, these methods augment the complexity of data processing and increase the workloads of field operation. Moreover, oblique photography is generally implemented manually to control oblique angles, which usually result in photographic gaps and weak intersection angles.

Cameras with novel design have also been proposed to address the problem of the limited format of single frames. Scanning photography is the photography approach in which the camera is controlled and rotated around one point to acquire images in multiple rows and columns to enlarge the FOV of one station. The images in numerous rows and columns are referred to as an image matrix. The core idea of scanning photogrammetry is that the view of stations can alternatively be enlarged given that the view of cameras is difficult to do so. This method is easier to exploit than to expand the format by multiple CCD detectors. In this approach, camera parameters, including focal length and pixel size of each projection, are kept constant 
as well $[24,25]$. A3 Edge, a contemporary aerial mapping camera developed and introduced by VisionMap, is an example of commercially available cameras for aerial photogrammetry. This camera takes images when it is rotated in a cross-track direction to enlarge FOVs [24]. In close-range measurements, a surveying robot carrying an image sensor and a theodolite or total station are typically applied, allowing the immediate acquisition of the position and posture of images [26]. In view of the limited image format used in surveying robots, theodolite scanning photogrammetry is proposed by controlling the robot to rotate the camera and acquire images in multiple rows and columns to enlarge the FOV of one station [27]. However, the use of surveying robots for measurements is expensive. Zhang et al. [16,28,29] proposed panning and multi-baseline photogrammetry to obtain rotating images during measurements. This photography approach specifies the overlaps among images, making it easy to organize images in order. Nevertheless, this technique is operated by hand, and only experienced photographers can accomplish the work well. L. Barazzetti et al. [30-32] generated gnomonic projective images from several pinhole images acquired by rotation platform with long focal lens to reconstruct a 3D model. This method provides a general network around the object using central perspective images with normal focal lens and reconstructs "sharp" details through gnomonic projections. Spherical panoramas have also been employed to extract 3D information [25,33-35]. G. Fangi [33] captured panoramas with the use of both normal and long focal lenses. The normal focal lens spherical panorama was used to establish the station coordinates, whereas the long one was employed to ensure accuracy.

In this study, we improve the solution of scanning photography. This method allows stations to be distributed in order as that in aerial photogrammetry and correspondingly allows standardized data to be acquired in close range. A photo scanner consisting of a non-metric camera, a rotation platform, and a controller is developed for convenient data acquisition without coverage gap. Once the operator sets the required parameters and specifies the ground coverage of the station, this machine can automatically control the camera to rotate in horizontal and vertical directions and obtain images as designed overlaps.

Our scanning photogrammetry method is expected to extract 3D information from long focal images immediately and robustly without the use of normal focal images to provide the general network. Therefore, we propose a modified triangulation according to the traits of data obtained by scanning photography. This developed approach can directly achieve high-precision measurement results regardless of the adopted focal lens (i.e., long or normal). Likewise, this approach is similar to processing images acquired with multiple CCD detectors. The main rationale underlying this technique is that the overlap between the ground coverages of adjacent image matrices is known and larger than $60 \%$ based on station distribution, whereas the overlap between stereo original images from different stations is unknown and may be less than $50 \%$. Thus, the relative orientation should be executed between the images synthesized from image matrices than among the original images. We then generate a free network of synthetic images and solve the exterior orientation elements of original images from elements of the former and their relative rotation angles recorded by the scanner. Consequently, we determine the overlap relationship among the original images. This method avoids identifying the overlap relationship among images by matching all of them to one another, which is a time-consuming approach. Finally, all of the original images are employed for final bundle adjustment because the multi-directional images obtained by scanning photography could affect the accuracy and robustness of the method. Considering the unknown interior elements and instable distortion in using DSLRs, we apply self-calibration bundle 
adjustment with control points to solve the interior elements of the camera; this approach has been adopted by most researchers [13,20,36,37].

The proposed method is applied in two test fields to validate the feasibility and precision of scanning photogrammetry. The experiments prove that scanning photogrammetry organizes image data well when large scenes are measured using long focal lens and achieves millimeter accuracy. Several comparative experiments are also performed to analyze the factors that may affect measurement accuracy.

The rest of this paper is organized as follows. Following the Introduction, Section 2 explains the theories of scanning photogrammetry. Section 3 evaluates the performance of the scanning photogrammetry system with the use of real datasets obtained with 300 and $600 \mathrm{~mm}$ lenses. Section 4 discusses the differences between our scanning photogrammetry technique and previous ones, and analyzes the reasons for the efficient measurement results. Finally, Section 5 presents the conclusions of the study and cites recommendations for future research.

\section{Proposed Scheme}

The proposed scheme comprises four parts, namely, Section 2.1 scanning photography, Section 2.2 station distribution, Section 2.3 scanning platform (photo scanner), and Section 2.4 data processing.

\subsection{Scanning Photography}

As previously mentioned, the size of a single image sensor is limited, thereby restricting the FOV of DSLRs when long focal lenses are used to yield comparatively high ground resolution images. Therefore, scanning photography was developed. This photography approach obtains sequences of images by rotating the camera in both horizontal and vertical directions to enlarge the FOV of one station. This technique is similar to VisionMap A3 camera, which acquires the flight sequences of frames in a cross-track direction to provide wide angular coverage of the ground [24]. When used in close-range photogrammetry, the presented scanning photography further improves traditional photography by exposing camera frames in both horizontal and vertical directions because the height of the camera is generally difficult to change in a wide range when high targets are photographed. This particular mechanism is similar to the concepts of "cross-track" and "along-track" in aerial photography. Each image captured by the camera is called an original image, and all original images taken at one station form an image matrix as displayed in Figure 1.

In scanning photography, rotation angles are calculated based on the principle that the relative rotation angles between two adjacent images in both directions are equal. However, in our experiments, this approach is inadequate because the images at the corners of image matrices may be covered by invalid areas as a result of the central perspective when photographing. Figure 2 illustrates how this scenario occurs. In this figure, points $\mathrm{D}, \mathrm{E}$, and $\mathrm{S}$ are in a horizontal plane, whereas points $\mathrm{A}, \mathrm{B}, \mathrm{C}, \mathrm{D}$ and $\mathrm{E}$ are in another plane that is perpendicular to the horizontal one. Point $\mathrm{B}$ is in line $\mathrm{AC}$, and $A D \perp D E$, $B E \perp D E, S E \perp D E$, and $S E \perp B E$. Angles $\angle B S E$ and $\angle A S D$ are defined as $\beta_{1}$ and $\beta_{2}$, respectively. Therefore, $\beta_{1}>\beta_{2}$. Points A, B, C, D, and E represent the center points of images A, B, C, D, and E, respectively. Image $\mathrm{E}$ is the reference image in both horizontal and vertical directions and is named the "normal" image. $\beta_{1}$ denotes the vertical angle rotating from images $E$ to $B$, and $\beta_{2}$ depicts the vertical angle rotating from images $\mathrm{D}$ to $\mathrm{A}$. Correspondingly, when photos are taken at the same height, the 
relative vertical rotation angle between the adjacent image rows far away from the "normal" image is smaller than that between the image rows near the "normal" image. Invalid coverages appear when the relative rotation angle in the vertical direction remains constant. This condition also applies to the rotation angle in the horizontal direction.

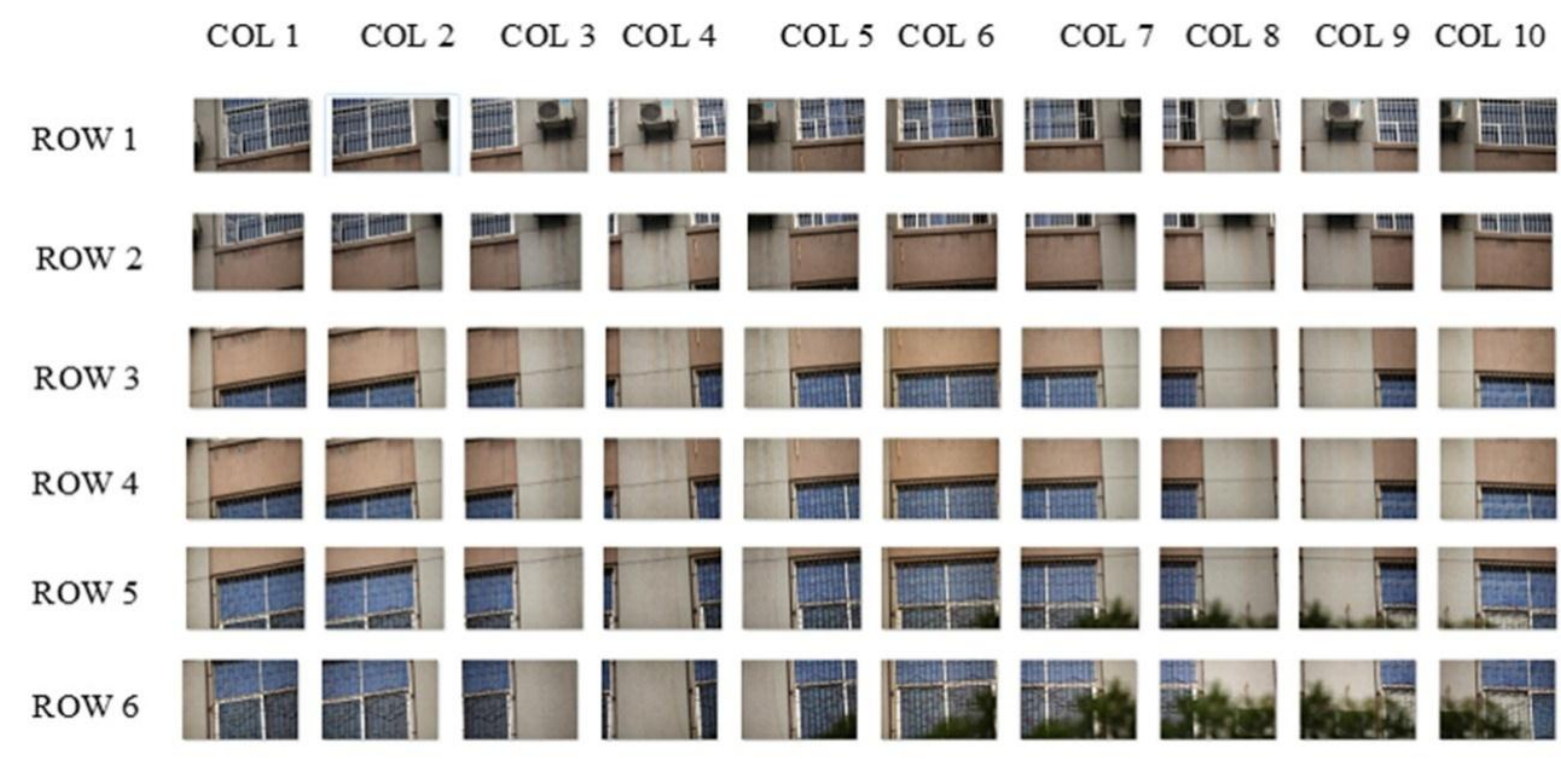

Figure 1. Illustration of image matrix at one station (The matrix contains 6 rows and 10 columns).

In view of the abovementioned problem, we propose an improved means for calculating rotation angles. Figure 3 presents how rotation angles are calculated using this method. $S$ is the perspective center. Horizontal rotation angle ranges from $\alpha_{1}$ to $\alpha_{2}$, and vertical rotation angle varies from $\beta_{1}$ to $\beta_{2}$. $f$ is the focal length, and region $W \times H$ is the ground coverage of the station projected to the focal plane of the "normal" image. The rotation angle of each original image is determined based on the designed overlaps in both directions. In Figure 3, the junctions of grids represent the image centers, and the rays from the perspective center to the image center indicate the principal rays of each image. The mechanism for calculating rotation angles is cited below. By following these procedures, we can calculate the rotation angles of images as in Equation (1).

(1) Obtain the range of rotation angles of a station. As mentioned above, horizontal rotation angle ranges from $\alpha_{1}$ to $\alpha_{2}$, and vertical rotation angle varies between $\beta_{1}$ and $\beta_{2}$.

(2) Region $W \times H$ can be calculated according to the angle range.

(3) Calculate the temporary horizontal and vertical distances between centers of the adjacent images in the same row and column in image matrix as $\Delta W_{\text {temp }}$ and $\Delta H_{\text {temp }}$, respectively. Accordingly, determine the row and column number of image matrix as $N_{\text {row }}, N_{c o l}$, respectively.

(4) Recalculate the horizontal and vertical distances as $\Delta W$ and $\Delta H$, respectively, to divide the region equally into rows $N_{\text {row }}$ and columns $N_{\text {col }}$.

(5) Determine the location of each image center on focal plane of the "normal" images, and calculate the rotation angles of each image in both directions. 
In our experiment, the absolute value of the horizontal and vertical rotation angles is assumed to be less than $60^{\circ}$. Figure 4 shows the horizontal and vertical rotation angles of original images in one image matrix; where the black solid dots denote the centers of images. In particular, the figure demonstrates that the relative rotation angle between adjacent images is decreased when the rotation angle increases from the "zero position." The improved results of scanning photography are depicted in Figure 5. To ensure successful measurement, the overlap between any two adjacent images in one image matrix should not be less than $30 \%$ in both directions.

To limit image distortion, the horizontal rotation angle $\alpha$ should be in the range of $\left[-45^{\circ}, 45^{\circ}\right]$, and the vertical rotation angle $\beta$ should be within $\left[-30^{\circ}, 30^{\circ}\right]$.

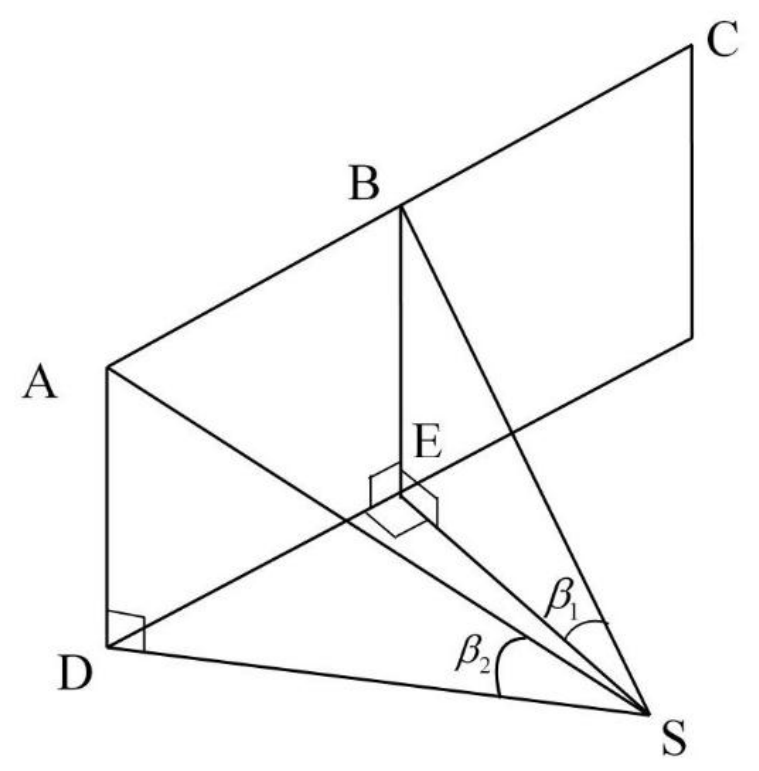

Figure 2. Rotation angles influenced as perspective projection.

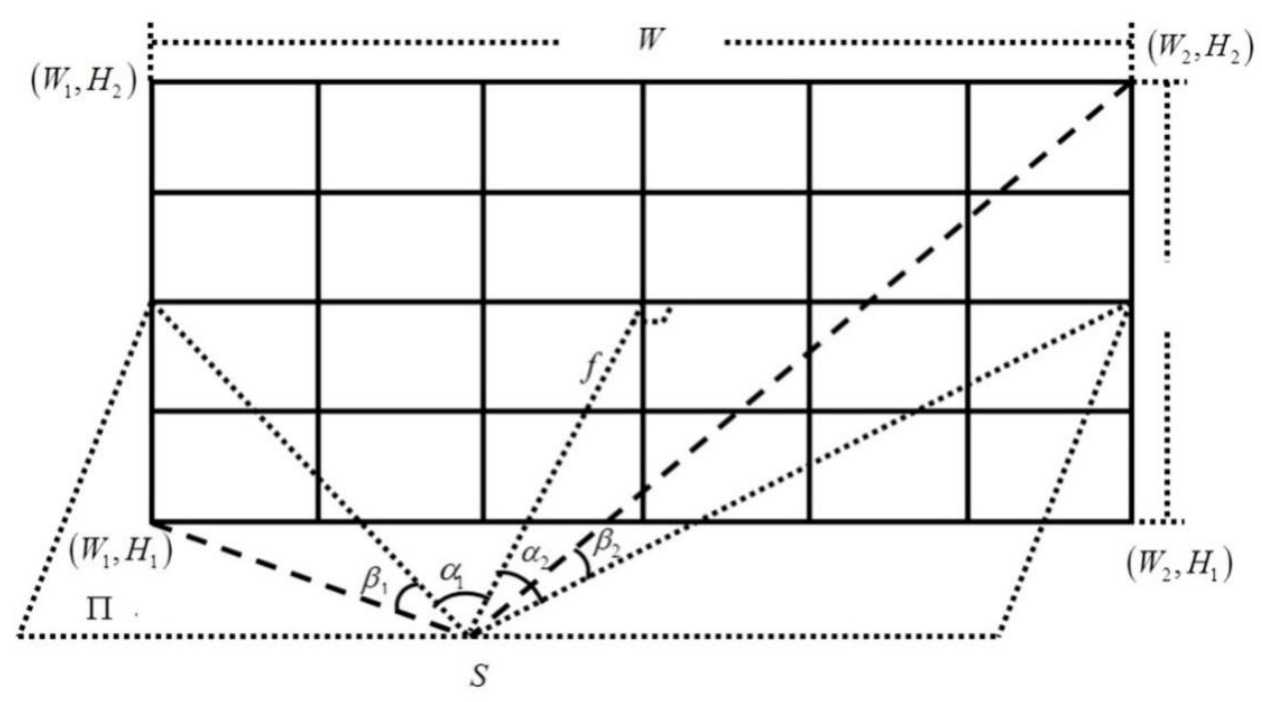

Figure 3. Calculation of rotation angles. 


$$
\begin{aligned}
& \Delta W_{\text {temp }}=\left(1-p_{x}\right) \cdot f \cdot 2 \tan \left(\phi_{H} / 2\right) \\
& \Delta H_{\text {temp }}=\left(1-p_{y}\right) \cdot f \cdot 2 \tan \left(\phi_{V} / 2\right) \\
& W_{1}=f \cdot \tan \alpha_{1} \quad W_{2}=f \cdot \tan \alpha_{2} \quad W=W_{2}-W_{1} \\
& H_{1}=\sqrt{f^{2}+W_{1}{ }^{2}} \cdot \tan \beta_{1} \quad H_{2}=H_{1}=\sqrt{f^{2}+W_{2}{ }^{2}} \cdot \tan \beta_{2} \quad H=H_{2}-H_{1} \\
& N_{\text {col }}=\left\lceil W / \Delta W_{\text {temp }}\right\rceil \quad N_{\text {row }}=\left\lceil H / \Delta H_{\text {temp }}\right\rceil \\
& \Delta W=W / N_{\text {col }} \quad \Delta H=H / N_{\text {row }} \\
& \alpha_{r c}=\tan ^{-1}\left(\left(W_{1}+c \cdot \Delta W\right) / f\right) \\
& \beta_{r c}=\tan ^{-1}\left(\left(H_{1}+r \cdot \Delta H\right) / \sqrt{\left(W_{1}+c \cdot \Delta W\right)^{2}+f^{2}}\right)
\end{aligned}
$$

where $f$ is the focal length; $p_{x}$ and $p_{y}$ are the known horizontal and vertical overlaps between images in the image matrix, respectively; $\phi_{H}$ and $\phi_{V}$ are the horizontal and vertical views of the camera, respectively; $\alpha_{1}$ and $\beta_{1}$ are the horizontal and vertical rotation angles of the image at the bottom left corner of the image matrix, respectively; $\alpha_{2}$ and $\beta_{2}$ are the rotation angles of the image at the top right corner; $W$ and $H$ are the width and height of the projected region on the "normal" image, respectively; $\Delta W_{\text {temp }}$ denotes the temporary horizontal distance between centers of the adjacent images in the same row in image matrix; $\Delta H_{\text {temp }}$ is the temporary vertical distance between centers of the adjacent images in the same column; $N_{\text {row }}$ and $N_{c o l}$ are the row and column numbers of the image matrix, respectively; $\Delta W$ represents the distance between adjacent image centers in the same row; $\Delta H$ is the final distance between adjacent image centers in the same column; and $\alpha_{r c}$ and $\beta_{r c}$ depict the horizontal and vertical rotation angles of the image at row $r$ and column $c$ in the image matrix, respectively.

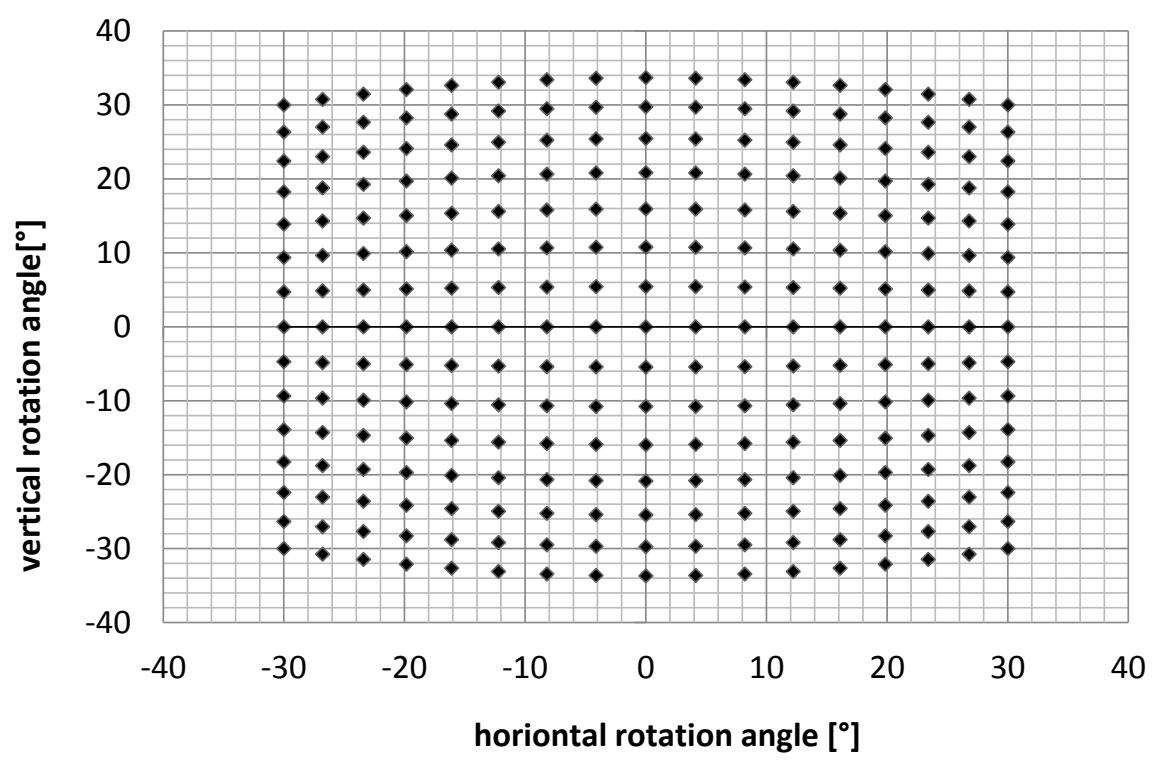

Figure 4. Distribution of horizontal and vertical rotation angles; the focal length is $100 \mathrm{~mm}$, and the camera format is $36 \mathrm{~mm} \times 24 \mathrm{~mm}$. The rotation angles of the image at the bottom left corner and top right corner of the image matrix are $\left(-30^{\circ},-30^{\circ}\right)$, and $\left(30^{\circ}, 30^{\circ}\right)$, respectively. The set overlaps in horizontal and vertical directions are $80 \%$ and $60 \%$, respectively. 


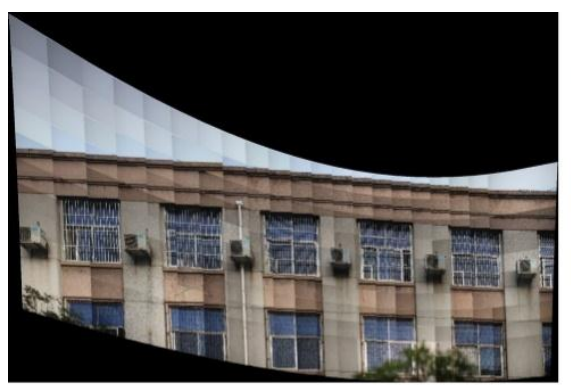

(a)

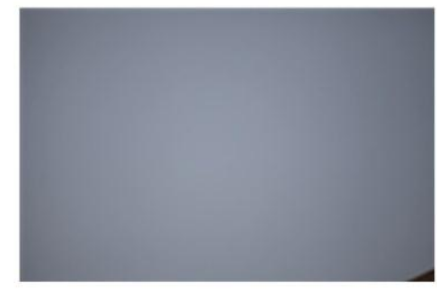

(c)

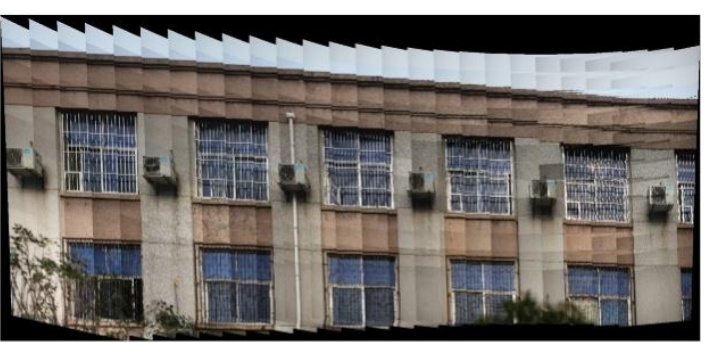

(b)

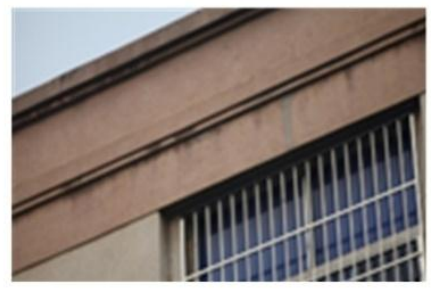

(d)

Figure 5. Synthetic images from image matrices with rotation angles calculated in different approaches; (a) is the synthetic image from the image matrix acquired using the same approach in determining the relative rotation angle between adjacent images; (b) is the synthetic image from the image matrix acquired with the improved method introduced in this paper; (c) is the image at the top left corner of the image matrix presented in Figure 5a; (d) is the image at the top left corner of the image matrix presented in Figure 5b.

\subsection{Station Distribution}

To facilitate data acquisition and processing, our station distribution is set similar to the flight strip in aerial photogrammetry. The top view of this distribution is displayed in Figure 6, where the horizontal line denotes the measured target, the rectangles represent the stations, and the corresponding two solid lines stretching from the station to the object are the FOV of the station. $S$ denotes the length of the target, and $D$ is the photographic distance (referred to as photo distance hereafter for brevity). For easy manipulation, the baseline denoted by $B$ between adjacent stations is designed the same, which is the same as aerial photogrammetry. Then, the operator will easily find out the location of the next station during data acquisition. To ensure the maximum utilization of images, the columns and rows of each station are varied because the FOV of each station is different. $\theta$ is the least FOV of stations determined by operators. $N$ represents the total number of stations, and $M$ is the given number of least stations from which any interest point on the target would be photographed. Figure 6a shows the station distribution when the target has a large width. In such a case, the distance from the first station to the last is the same as the width of the target. In our scanning photography mechanism, the FOV of stations ranges from $\theta$ to $2 \theta$ according to the location of the station. Similarly, the largest intersection angle of the measured points ranges from $\theta$ to $2 \theta$. We limit $\theta$ between $20^{\circ}$ and $45^{\circ}$ to maintain a good balance between measurement and matching precisions. Photo distance $D$ is determined according to the selected camera focus, required measurement precision, and scene environment. However, certain scenarios exist (e.g., Figure 6b) in which the target is extremely narrow such that the FOV of a station is greatly limited in the given photo distance. Figure $6 \mathrm{~b}$ depicts the solution for this condition, ensuring the intersection 
angle at measuring points. The distance from the first station to the last is larger than the width of the target. As such, the intersection angle at the measuring points is larger than $\theta$, and the view angle of stations can either be smaller or larger than $\theta$. To maintain measurement precision, every point on targets should be photographed from at least three stations. In other words, $M$ should be selected, but it should not be less than 3. Then, the overlap between two adjacent stations is set above $67 \%$ to enable stereo photogrammetric mapping. It is known that large overlap will result in small baseline. However, as in Figure 6, our scanning photogrammetry is a kind of photogrammetry method using multi-view images. Image correspondences of each tie point for bundle adjustment are from multiple stations, which can form considerable intersection angle. Even if the baseline of the adjacent stations is small, it will not affect the measurement precision. Nevertheless, a small baseline will lead to consuming more time for data obtainment and processing. Therefore, we advise the operators to determine the parameter after balancing the data acquisition time and variation of the target in the depth direction. These parameters are computed as in Equation (2).

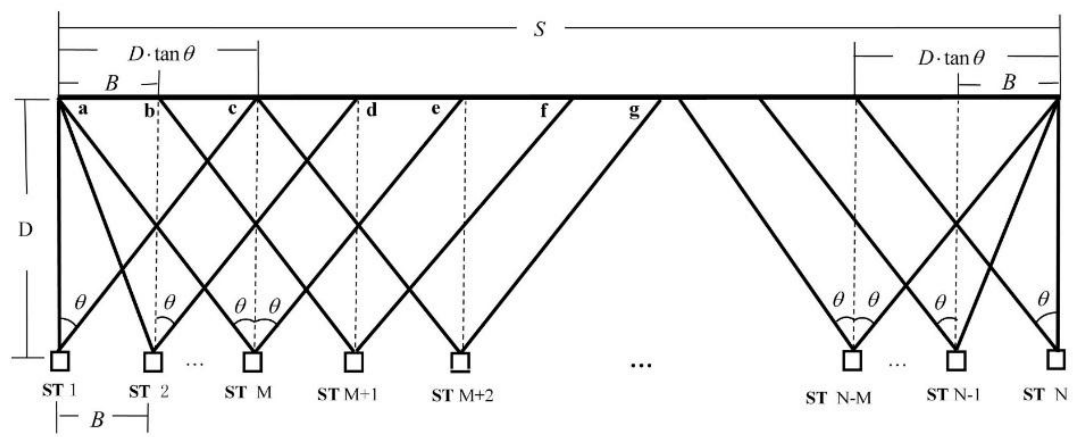

(a)

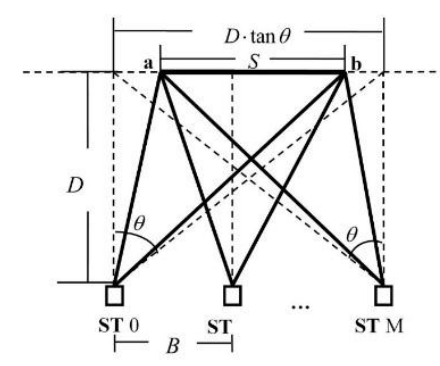

(b)

Figure 6. Station distribution: (a) station distribution of wide target measuring; (b) station distribution of narrow target measuring.

$$
\begin{aligned}
& B_{\text {temp }}=D \cdot \tan \theta /(M-1) \\
& N=\left\lfloor S / B_{\text {temp }}+0.5\right\rfloor+1 \\
& \left\{\begin{array}{c}
\text { if } N \geq M, \quad B=S /(N-1) \\
\text { if } N<M, \quad B=D \cdot \tan \theta \cdot(M-1), \quad N=M
\end{array}\right.
\end{aligned}
$$

where $B_{\text {temp }}$ and $B$ are the temporary and final baselines, respectively; $D$ is the photo distance $\theta ; \theta$ is the given least FOV of a station; $S$ is the length of the target; $M$ is the given number of least stations from which any interest point on the target would be acquired; and $N$ is the total number of stations.

Stations should be distributed in adherence to the following rules: 
(1) The first station should be aligned to the left edge of the target shown in Figure 6a. However, when faced with the situation as in Figure 6b, the first station should be located at the distance of $(D \cdot \tan \theta-S) / 2$, away from the left edge of the target.

(2) The photo distances of every station are approximately equal when the variation of the target in depth direction is not large. Otherwise, the stations should be adjusted to the variation of the target in depth direction to maintain equal photo distances.

(3) The length of the baselines should be the same.

After the location of the first station is identified, the location of the remaining stations can be determined according to the abovementioned principles. As demonstrated in Figure 6a, the horizontal coverage of the first, second, and third stations ranges from points a to c, a to d, and a to e, respectively. Contrarily, the fourth station covers points $b$ to $f$. The remaining stations follow the same concept. In Figure $6 \mathrm{~b}$, points $\mathrm{a}$ and $\mathrm{b}$ denote the beginning and end of the target, respectively, and the horizontal coverage of each station begins from point $a$ to $b$.

Since the height of the different parts of the target is variable, the vertical view of each station is based on the height of the photography region. Given that a large pitch angle leads to severe image distortion, the vertical rotation angle of each station should be limited in the range of $-30^{\circ}$ to $30^{\circ}$. In other words, when the target is extremely high, the height of the instrument or the photo distance must be increased to maintain the vertical view within range.

\subsection{Photo Scanner}

To guarantee image quality and measurement efficiency, we develop a photo scanner for automatic data acquisition. This scanner shown in Figure 7 consists of a non-metric camera, a rotation platform, a controller (e.g., PC or tablet PC with Win7/8), and a lithium battery. The camera is mounted on the rotation platform composed of a motor unit and transmission mechanism; this platform controls the camera scanning across horizontal and vertical directions. Figure 7 indicates that the rotation center of the platform is not the perspective center of the camera. However, when the scanner is used for large engineering measurements, the offset values are relatively tiny compared with photo distance. A module for determining rotation angles is also integrated into the software to transmit data and control platform rotation. The scanner automatically obtains the image once the camera parameters (i.e., focus and image format), data storage path, horizontal and vertical overlaps between images in each image matrix, and ground coverage of stations are inputted into the software by the operators. For convenient measurement, the scanner is designed to be mounted on a tripod for the total station, and the battery unit for the rotation platform can be hung on the tripod. Thus, the developed photo scanner can be carried and operated by only one person when conducting measurements, providing great convenience.

The mechanism for applying the developed scanner is as follows:

(1) The instrument is installed and placed at the proper position.

(2) The photographic plane is paralleled to the average plane of the needed photographing region, and the rotation platform is leveled with the bubble on the instrument base. This position of the camera is defined as the "zero posture" in this station and is considered the origin of rotation angles in both horizontal and vertical directions. 
(3) The FOV of this station is specified, and the required parameters are inputted into the controller.

(4) The number of rows and columns of the image matrix as well as the rotation angles in horizontal and vertical directions for each image are computed, and the signal is sent to manage the rotation and exposure of the camera for automatic image acquisition.

Data can be stored by transferring images into the controller (e.g., PC or tablet PC) and storing them in a compact flash card. In the second method, the images from the camera are not required to be transmitted to the PC or tablet PC. As such, the photo interval is shorter. During photographing, the controller enables a real-time quick view of the captured images. At the same time, the rotation angles of each captured image are stored in the controller as a log file, which can be used as auxiliary information for data processing.

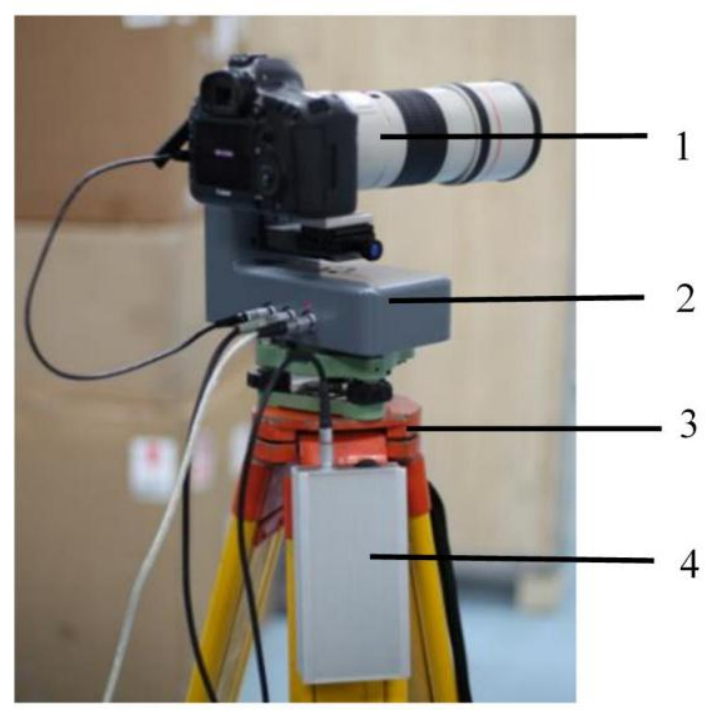

1. non-metric camera

2. rotation platform

3. tripod

4. lithium battery

Figure 7. Parts of the photo scanner

\subsection{Data Processing}

In our proposed method, data are automatically processed once the user specifies the data directory and required outputs. To achieve high accuracy and stable solution, all original images are used for bundle adjustment because redundant observations are helpful in obtaining a robust solution, and information loss is unavoidable during image synthesis. Considering that all original images are needed for bundle adjustment, we employ modified triangulation to improve the efficiency of data organization and processing. As previously noted, the core idea of modified triangulation is that although the overlaps between original images from different stations remain unknown, we can estimate the overlaps between coverages of image matrices according to the station distribution. Thus, we use the images synthesized from the image matrix to generate a free network. We then compute the initial exterior elements of original images in this free network from the elements of synthetic images and rotation angles of original images. After the matching relationships among the original images from neighboring image matrices are identified according to the matching results of the two corresponding synthetic images, image matching is executed among original images to determine tie points. Self-calibration bundle adjustment with control points is then performed. In establishing the initial exterior parameters of original images, 
we assume that the perspective centers of original images and their corresponding synthetic images are the same even though they are actually different. The accurate exterior parameters of original images that vary from those of the corresponding synthetic images can be obtained after bundle adjustment. Figure 8 exhibits the flow of data processing. The processes of taking original images with long focal lens into bundle adjustment are determined to be similar to that using the VisionMap A3 system. Thus, this procedure is valid and can be used in both aerial and close-range photogrammetry.

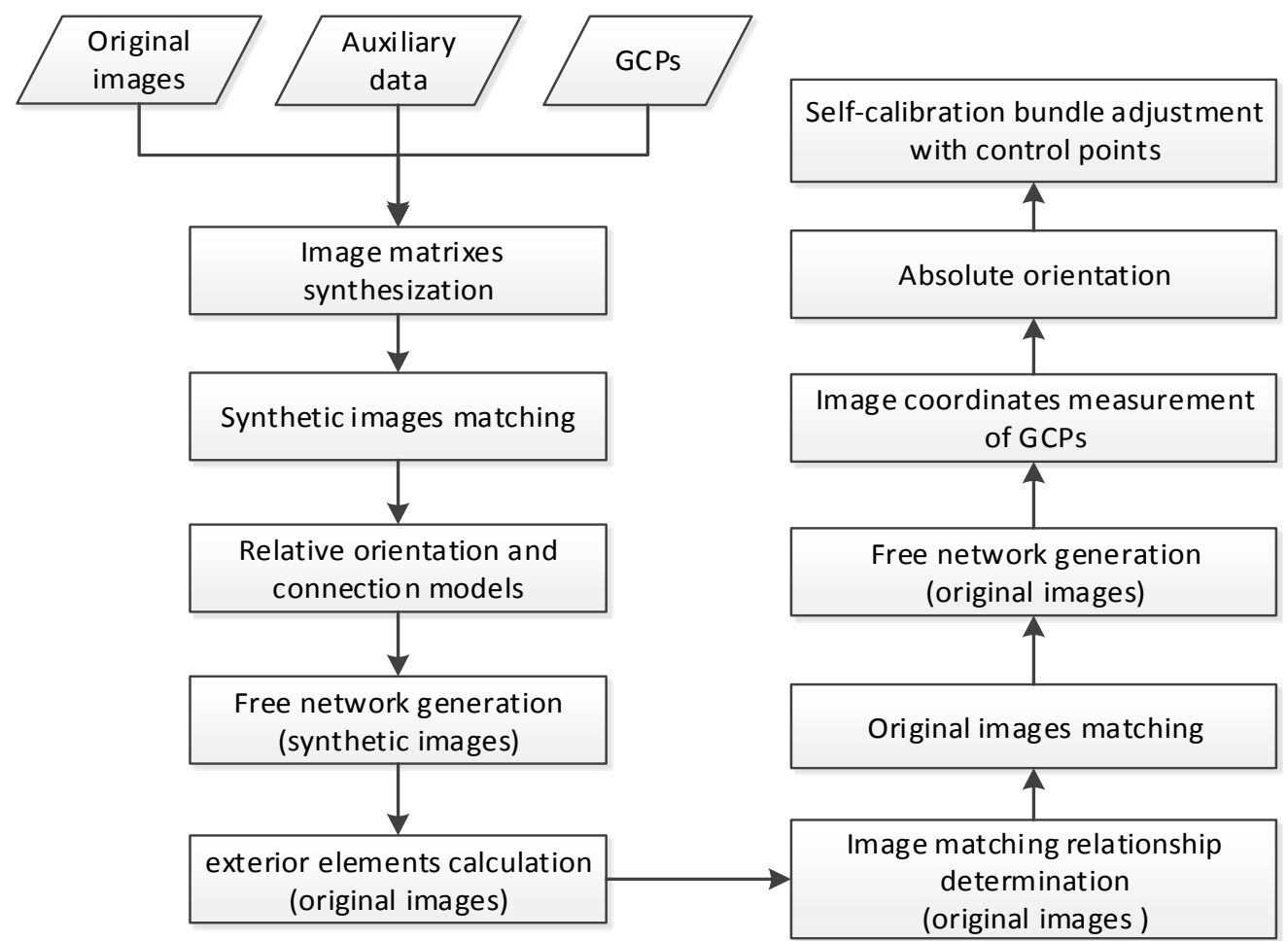

Figure 8. Flow chart of data processing

\subsubsection{Synthetic Image}

In practical measurements in scanning photography, the unknown overlaps between stereo pairs of original images from different stations are not always large enough for relative orientation. This condition therefore leads to an unstable relative orientation among stereo pairs. Considering that the overlaps between station image matrices are large enough for relative orientation, we apply a simplified method of synthesizing image matrices for free network generation. In view of information loss when original images are synthesized from image matrices, synthetic images are not used for final bundle adjustment but are only used for computing the initial exterior elements of original images. Although the camera is not rotated around the perspective center, the offset values from its center to the center of rotation are relatively tiny compared with the photo distance when performing large engineering measurements using the developed photo scanner. Therefore, we simplify the model by ignoring the offset values and projecting the image matrix to the focal plane of the "normal" image to synthesize the image matrix. Figure 9 illustrates the image synthesis model. For simplicity, only one row of the image matrix is graphed. The rotation angles of the images can be presented as $\left(H_{i}, V_{i}\right)(i=1,2, \ldots, m) . H_{i}$ and $V_{i}$ are the horizontal and vertical angles, respectively, and $m$ is the number of images in the image 
matrix. The method used to generate a synthetic image is to re-project all original images in the image matrix to the equivalent focal length plane with $H=0$ and $V=0$, as shown in Figure 9. Equation (3) is employed to obtain synthetic images given that the scanner is designed to rotate first in the horizontal direction and then in the vertical direction.
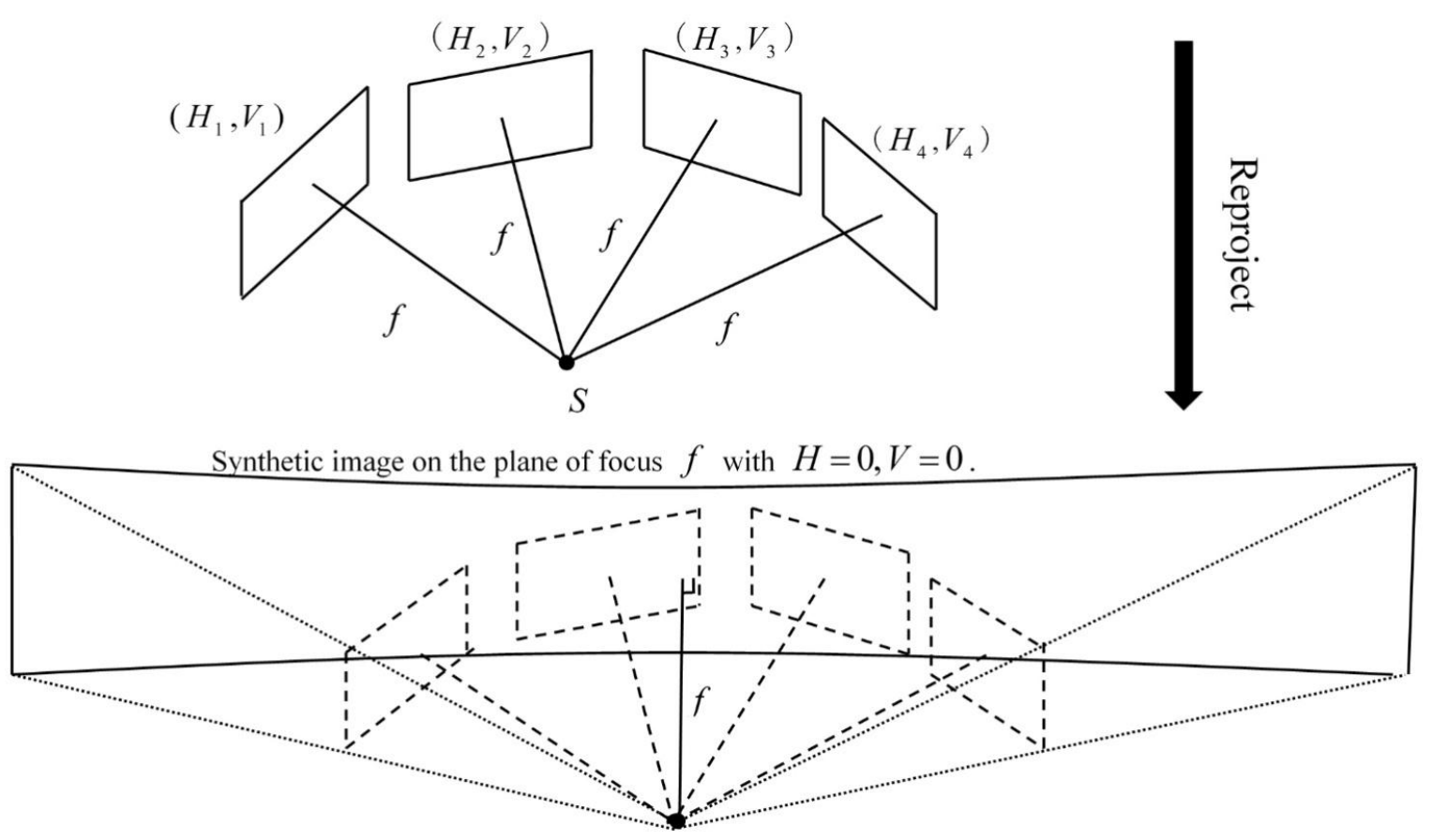

$S$

Figure 9. Model of image synthesis

$$
\begin{gathered}
{\left[\begin{array}{c}
x^{\prime} \\
y^{\prime} \\
-f
\end{array}\right]=R_{i}^{H V}\left[\begin{array}{c}
x_{i} \\
y_{i} \\
-f
\end{array}\right]} \\
R_{i}^{H V}=\left[\begin{array}{ccc}
\cos H_{i} & 0 & -\sin H_{i} \\
0 & 1 & 0 \\
\sin H_{i} & 0 & \cos H_{i}
\end{array}\right] \cdot\left[\begin{array}{ccc}
1 & 0 & 0 \\
0 & \cos V_{i} & -\sin V_{i} \\
0 & \sin V_{i} & \cos V_{i}
\end{array}\right]
\end{gathered}
$$

where $x$ and $y$ denote the image coordinates in image $i, x^{\prime}$ and $y^{\prime}$ are the coordinates in the synthetic image, and $R_{i}^{H V}$ is the rotation matrix from the synthetic image to frame $i$.

The synthesis method explained above is suitable only when the view of the image matrix is not significantly large $\left(\leq 120^{\circ}\right)$.

\subsubsection{Image Matching and Error Detection}

In general, the parallax may change in a wide range and be volatile because of occlusions in closerange image pairs. When the relative rotation angle is larger than $15^{\circ}$, which commonly occurs in oblique photography, traditional image matching is unsuitable. Alternatively, scale-invariant feature transform (SIFT) match can be steadily applied in many cases when it involves an angle that is less than $50^{\circ}$ [38]. Given that the SIFT features presented by 128-dimensional vectors are used for image matching, a long computational time is needed when a large number of feature points are involved. Thus, graphics 
processing unit (GPU) acceleration is employed. Considering that the memory of GPUs is limited, we perform a block-matching algorithm. In this algorithm, we simply use each block from one image to match all of the blocks from another image. The block matching results are accepted only when the number of correspondences is larger than the threshold.

Mismatching inevitably occurs in image matching methods. Thus, we should pay attention to error detection. The outliers in SIFT point matches are generally discarded with random sample consensus (RANSAC) for estimating model parameters. In our scanning photography, two kinds of error detection can be used after image matching, and the method to be applied is selected based on whether the corresponding points are from the same station. For matching images from the same station, the object coordinates of perspective centers are approximately equal. Therefore, the homography model [39] in Equation (4) is used as the estimation model. The geometric distortions between images are severe when the matching images are acquired from different stations, suggesting that transformation models (e.g., affine and projective transforms) are no longer suitable for detecting matching errors [40]. After performing several experiments, we adopt the quadric polynomial model in Equation (5) to estimate geometric distortions. This model is the superior choice in terms of computational time and measurement precision. The threshold of residual for RANSAC should be relaxed because the interior elements of original images are inaccurate, and the estimation model is not rigid.

$$
\left[\begin{array}{c}
x^{\prime} \\
y^{\prime} \\
1
\end{array}\right]\left[\begin{array}{lll}
h_{1} & h_{2} & h_{3} \\
h_{4} & h_{5} & h_{6} \\
h_{7} & h_{8} & h_{9}
\end{array}\right] \cdot\left[\begin{array}{l}
x \\
y \\
1
\end{array}\right]
$$

where $(x, y)$ and $\left(x^{\prime}, y^{\prime}\right)$ are the image coordinates on the left and right images in an image pair, respectively, $h_{1}, h_{2}, \ldots, h_{9}$ are elements of the homography matrix, and $h_{9}=1$.

$$
\begin{aligned}
& x^{\prime}=a_{1} x^{2}+a_{2} x y+a_{3} y^{2}+a_{4} x+a_{5} y+a_{6} \\
& y^{\prime}=b_{1} x^{2}+b_{2} x y+b_{3} y^{2}+b_{4} x+b_{5} y+b_{6}
\end{aligned}
$$

where $a_{1}, a_{2}, \ldots, a_{6}$, and $b_{1}, b_{2}, \ldots, b_{6}$ are quadric polynomial coefficients.

\subsubsection{Modified Aerial Triangulation}

The overlaps between synthetic images are known based on station distribution. Therefore, these images, instead of the original ones, are more suitable for interpreting epipolar geometry. The reason is that the overlaps among original images are unknown before the pre-processing of image matching. Thus, we use synthetic images in the triangulation of relative orientation and model connection to generate a free network. In close-range photogrammetry, the initial elements of relative orientation are crucial to achieving good solution in triangulation when intersection angles are large. In this event, a stable algorithm of relative orientation is required. Compared with other direct methods (e.g., 6-, 7-, and 8-point methods), the 5-point method performs best in most cases [41]. For this reason, we apply the 5-point relative orientation algorithm proposed by Stewenius [42] from the perspective of algebraic geometry. This algorithm employs a Gröbner basis to easily explain the solution [42]. To improve precision, an iterative scheme for refining relative orientation is adopted after the calculation of initial parameters. Each successive model is then connected with one another similar to the case in aerial triangulation. Correspondingly, the free network of synthetic images is established. 
The free network provides the exterior orientation elements of synthetic images. However, the exterior orientation elements of each original image are the required parameters for final bundle adjustment. Given that the changes are small among the space coordinates of each original image perspective center in one image matrix, the initial space coordinates of the original image are considered similar to those of their corresponding synthetic image. Accordingly, we can solve the azimuth elements of original images by decomposing the rotation matrix calculated with the azimuth elements of synthetic images and rotation angles of original images as depicted in Equation (6).

$$
R_{j i}=R_{j} \cdot R_{j i}^{H V}
$$

where $R_{j i}$ is the rotation matrix of image $i$ in station $j, R_{j}$ is the rotation matrix of synthetic image $j$ in the free network, and $R_{j i}^{H V}$ is the rotation matrix of relative rotation angles from the synthetic image of station $j$ to image $i$.

Before bundle adjustment with all original images, image matching should be executed among original images to obtain the corresponding points as tie points for bundle adjustment. Stereo matching is performed between the original images from different stations as well as between the original images from the same station. We find six images, namely, two from the same station and four from the next station, which overlap with each original image to proceed with matching. For the images from the same station, we chose the two images at the right of and below the processing image in the image matrix. Meanwhile, for the images from the next station, we chose four images with the highest overlapping rate with the image for stereo matching. The overlapping rate is calculated according to the matching results of the two corresponding synthetic images. Then, we tie all matching results together and then use multi-image forward intersection to compute the space coordinates of the tie points in the free network. As a result, the free network of original images is established. Absolute orientation is performed to establish the relationship between the space coordinate system of free network and the object space coordinate system before bundle block adjustment. We then compute the exterior elements of original images and space coordinates of tie points in the object space coordinate system.

In general, traditional bundle adjustment is the most mature approach because the collinearity condition for all tie points (TPs) and ground control points (GCPs) are satisfied simultaneously [43]. Considering the instability of intrinsic parameters of non-metric cameras, we utilize the on-line self-calibration bundle adjustment with control points to obtain the final result. In consideration of the camera intrinsic parameters, radial distortion, and tangential distortion, the mathematical model of self-calibration bundle adjustment based on the collinearity equation is established as in Equation (7) [44].

$$
\begin{aligned}
& x-x_{0}-\Delta x=-f \frac{a_{1}\left(X-X_{S_{i}}\right)+b_{1}\left(Y-Y_{S_{i}}\right)+c_{1}\left(Z-Z_{S_{i}}\right)}{a_{3}\left(X-X_{S_{i}}\right)+b_{3}\left(Y-Y_{S_{i}}\right)+c_{3}\left(Z-Z_{S_{i}}\right)} \\
& y-y_{0}-\Delta y=-f \frac{a_{2}\left(X-X_{S_{i}}\right)+b_{2}\left(Y-Y_{S_{i}}\right)+c_{2}\left(Z-Z_{S_{i}}\right)}{a_{3}\left(X-X_{S_{i}}\right)+b_{3}\left(Y-Y_{S_{i}}\right)+c_{3}\left(Z-Z_{S_{i}}\right)}
\end{aligned}
$$

where

$$
\begin{gathered}
\Delta x=\left(x-x_{0}\right)\left(k_{1} r^{2}+k_{2} r^{4}\right)+p_{1}\left[r^{2}+2\left(x-x_{0}\right)^{2}\right]+2 p_{2}\left(x-x_{0}\right)\left(y-y_{0}\right) \\
\Delta y=\left(y-y_{0}\right)\left(k_{1} r^{2}+k_{2} r^{4}\right)+p_{2}\left[r^{2}+2\left(y-y_{0}\right)^{2}\right]+2 p_{1}\left(x-x_{0}\right)\left(y-y_{0}\right) \\
r^{2}=\left(x-x_{0}\right)^{2}+\left(y-y_{0}\right)^{2}
\end{gathered}
$$


where $(x, y)$ is the image coordinate of a point in image $i ;[X, Y, Z]$ is the object coordinate of the point; $\left[X_{S_{i}}, Y_{S_{i}}, Y_{S_{i}}\right]$ is the object coordinate of perspective center of image $i ; a_{1}, a_{2}, a_{3}, b_{1}, b_{2}, b_{3}, c_{1}, c_{2}$, and $c_{3}$ are elements of the rotation matrix of image $i$; $f$ is the focal length; $\left(x_{0}, y_{0}\right)$ denotes the coordinate of the principal point of image $i ;(\Delta x, \Delta y)$ denotes the correction of the lens distortion; $k_{1}$ and $k_{2}$ are radial distortion coefficients; and $p_{1}$ and $p_{2}$ are tangential distortion coefficients.

During bundle adjustment, we can establish several correspondence pairs for each tie point, and the corresponding points can be either from the same station or from different stations. The pairs from the different stations are characterized by large intersection angles that are beneficial to the accuracy of depth direction, whereas the pairs from the same station are characterized by very small intersection angles that may lower accuracy. Thus, we consider the observations of image coordinates of tie points as correlative rather than independent, and the correlation coefficient is inversely proportional to the intersection angle. The weight of observations is based on intersection angles as considered in bundle adjustment and the weight of the ground points is set to 10 times the tie points. Iterative least squares adjustment is exploited for solving the unknown parameters of original images. Finally, accurate solutions are yielded for all images.

\section{Experimental Results}

\subsection{Experimental Data}

Scanning photogrammetry has been proposed mainly for acquiring data when large targets are measured and for obtaining accurate measurements of large objects at any focal length. Here, we emphasize in our experiments the application of cameras with long focal lens. Our method has been proven effective in experiments involving measuring the flag platform of Wuhan University and the high slope of Three Gorges Project permanent lock, as shown in Figure 10. The Cannon 5D Mark II camera (format size: $5616 \times 3744$, pixel size: $6.4 \mu \mathrm{m}$ ) is used for the tests. In the experiments, reflectors for the total station are used as control points. Object surfaces will cause reflectors to be attached unevenly because the surfaces may be rough. We use plexiglass boards, which are thicker, as the baseplates of reflectors to ensure that the reflectors attached to the object surfaces are even. Figure 10a shows a quick view of the flag platform. The height, width, and depth of this area are 10, 35, and $12 \mathrm{~m}$. As shown in the figure, there are stairways leading to the flag platform from the playground, resulting in several layers in the measurement area, which brings difficulty in data processing because of the discrete relief displacement. Control points are measured by Sokkia SET1130R3 total station with $3 \mathrm{~mm}$ accuracy. Figure 10b shows a quick view of the high slope of Three Gorges Project permanent lock. The height, width, and depth of this region is 70,150 , and $70 \mathrm{~m}$. Owing to the special environment in this field, the best position for measurement is the opposite side of the lock. The photo distance can reach up to $250 \mathrm{~m}$. The Leica TCRA1201 R300 total station is used to measure control points with an accuracy of $2.9 \mathrm{~mm}$. 


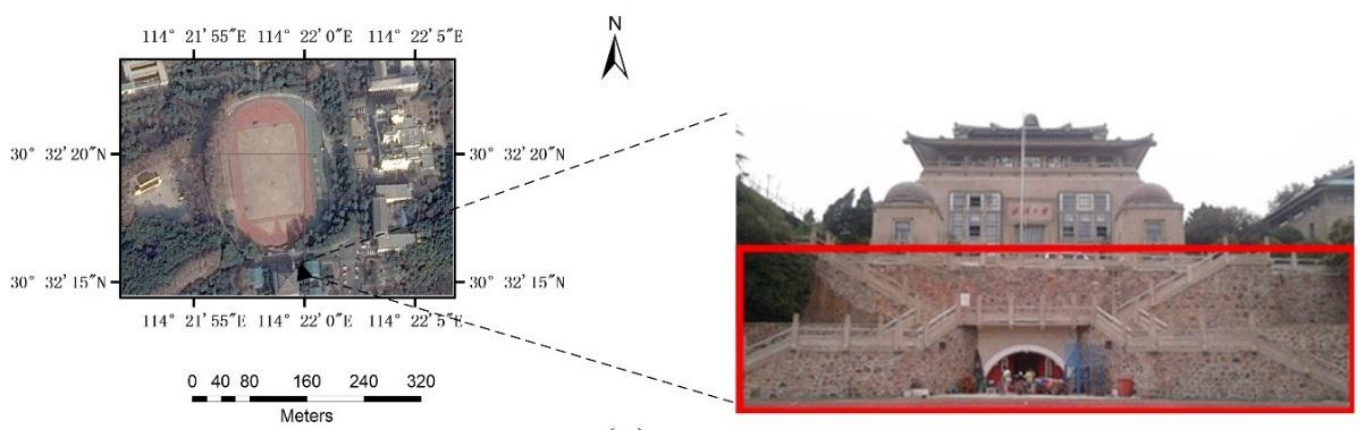

(a)

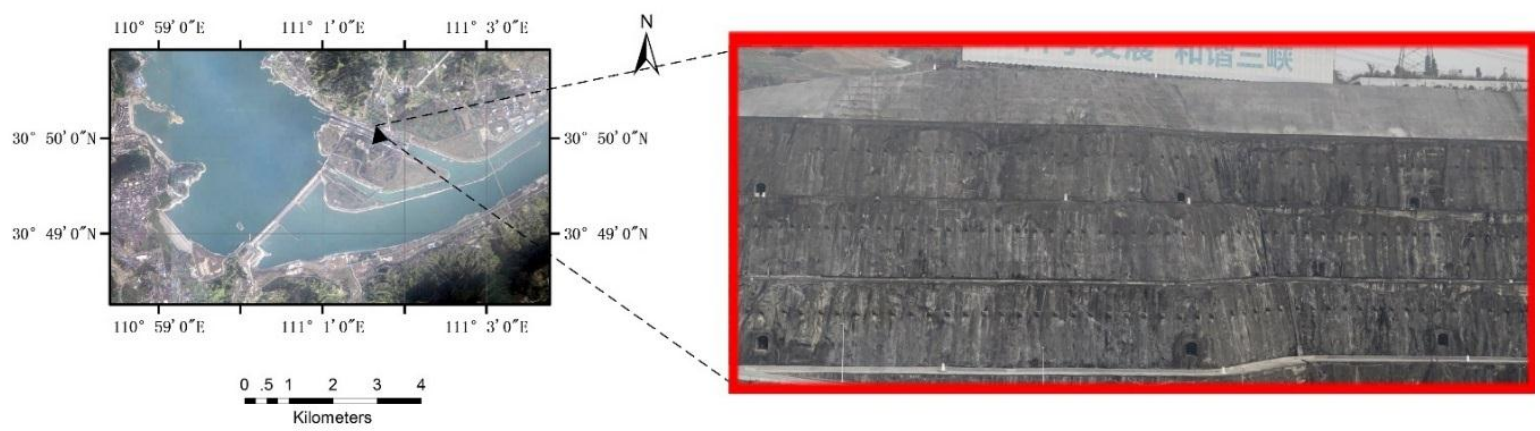

(b)

Figure 10. Quick view of test fields. ((a) flag platform of Wuhan University.; (b) high slope of Three Gorges Project permanent lock. The regions in the red rectangles show the measuring ranges.)

\subsection{Results of Synthetic Images}

Figure 11 illustrates the synthetic images of the two test fields. Figure 11a shows the synthetic image of one station for the field test of the flag platform with $300 \mathrm{~mm}$ focus lens, and the photo distance is 40 $\mathrm{m}$. Figure $11 \mathrm{~b}$ shows the synthetic image of one station for test field of the high slope of Three Gorges Project permanent lock with $300 \mathrm{~mm}$ focus lens at a distance of $250 \mathrm{~m}$. The method applied to yield synthetic images is described in Section 2.4.1. As shown, the invalid area in the synthetic images is slightly due to the use of the improved method. The performance is not that good, because no post-processes, such as unifying color or finding best seam lines, are executed. However, these factors do not have much influence on the matching of synthetic images.

\subsection{Results of Image Matching (Synthetic Images and Original Images)}

Three kinds of image matching exist in this process: one for synthetic images, one for original images from different stations, and one for original images from the same station. For synthetic images, every two overlapping images is a stereo pair. However, for original images, only two overlapping images from different stations can form a stereo pair. Both stereo pairs and non-stereo pairs are needed to be matched with the SIFT matching method. After SIFT matching is performed, error detection methods are used to refine the matching results. Depending on whether the image pair is a stereo pair or a non-stereo pair, RANSAC with estimation by quadric polynomial or homography model is used for detecting errors. 
Figures 12 and 13 show the results of matching and mismatching points in synthetic images from two stations. The red crosses denote the detected correct corresponding points, whereas the blue crosses denote the detected errors. The detection model is a quadric polynomial model. The matching results of the original images are shown in Figures 14-17. Image pairs in Figures 14 and 16 are stereo pairs and are processed by quadric polynomial models. Meanwhile, image pairs in Figures 15 and 17 are non-stereo pairs and are processed by homography models. The results verify that these error detection methods are valid. Given that an amount of feature points are extracted from the images showed in Figures 14 and 15, we perform block-matching algorithm. Thus, matching errors clustering exists because of the mechanism of our block-matching algorithm. However, our error detection method can eliminate outliers efficiently.

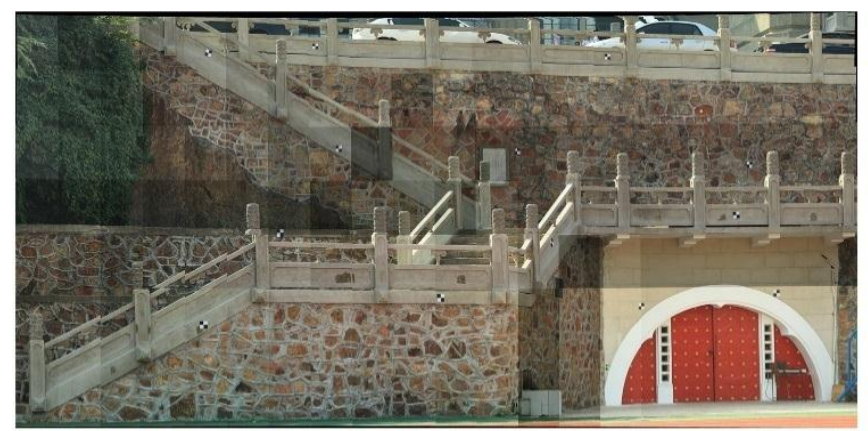

(a)

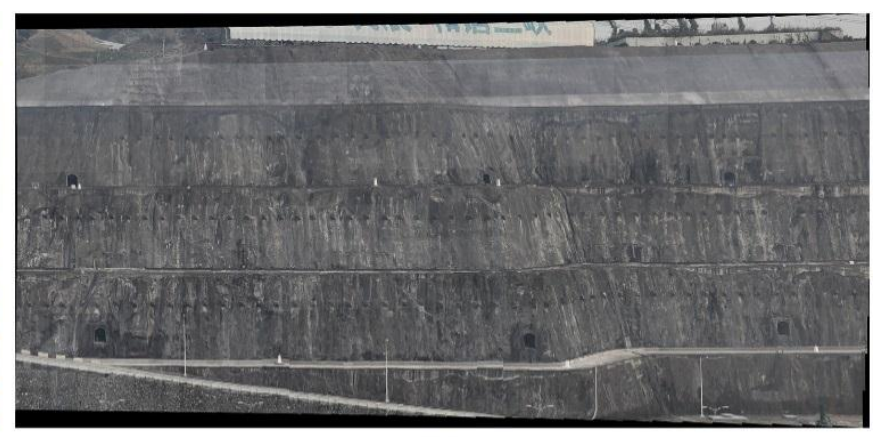

(b)

Figure 11. Synthetic images: (a) synthetic image of one station for test field showed in Figure 10a; (b) synthetic image of one station for test field showed in Figure 10b.

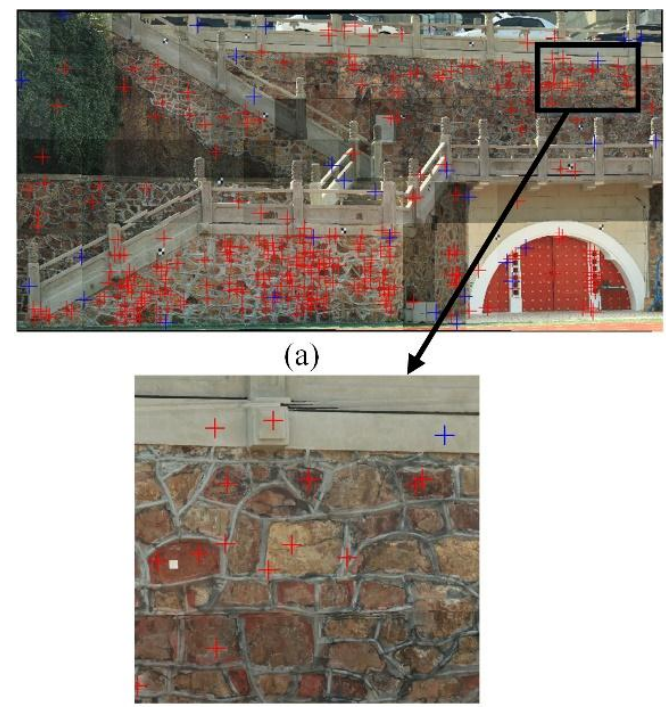

(c)

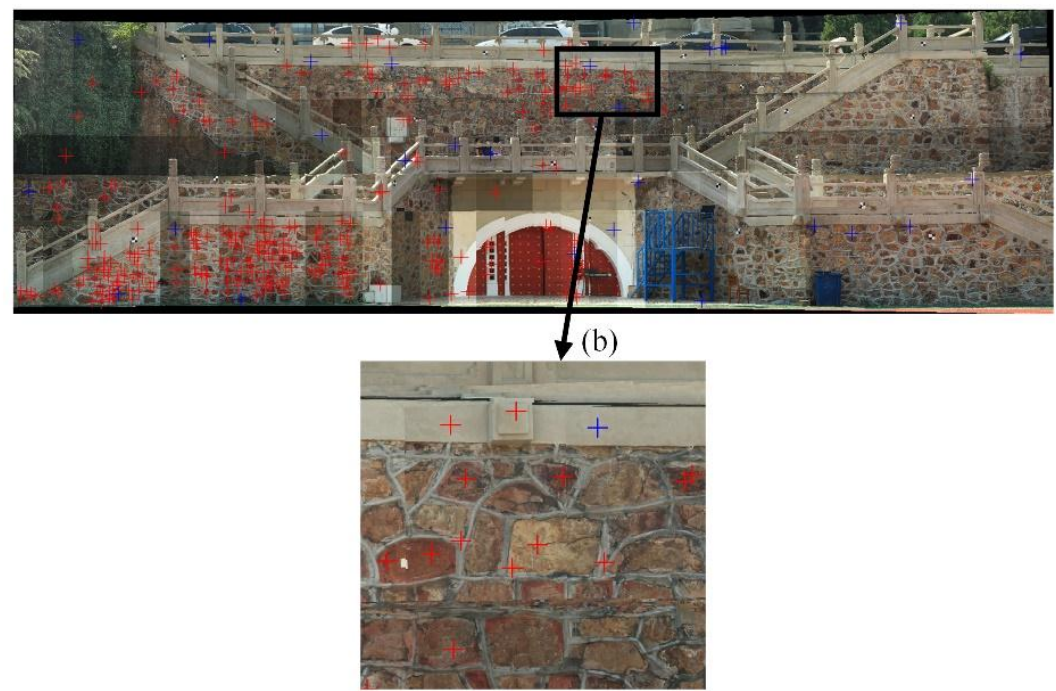

(d)

Figure 12. Synthetic images matching. (a,b) shows the matching results of synthetic images from the first and second station for test field in Figure 10a; (c,d) illustrates part of the results. 


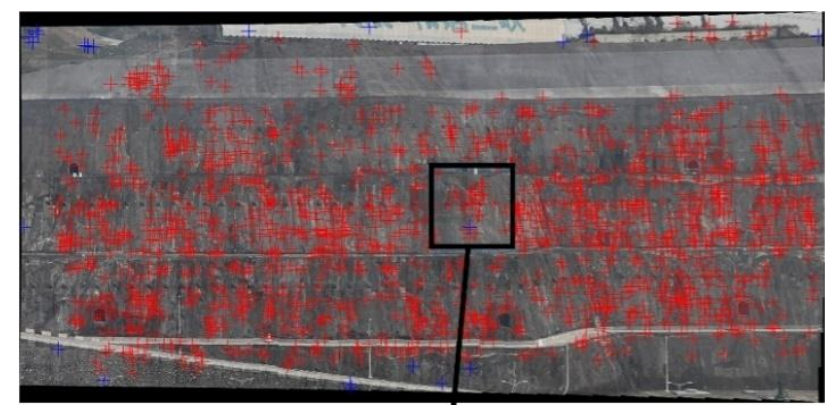

(a)

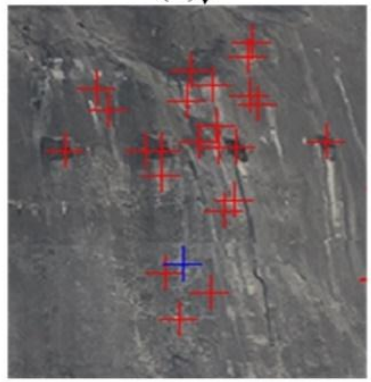

(c)

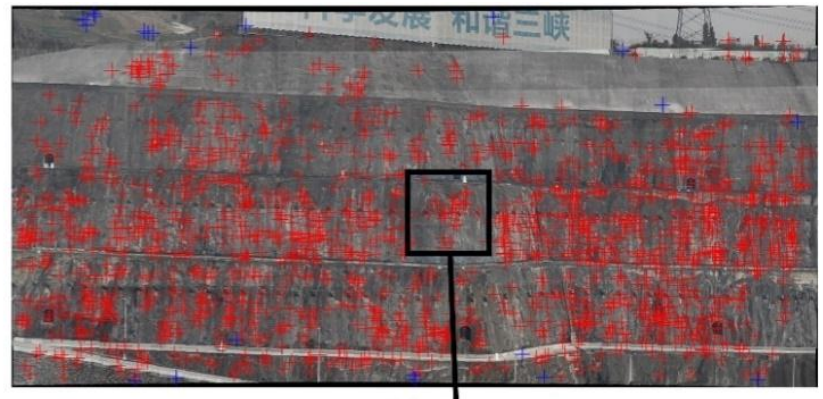

(b)

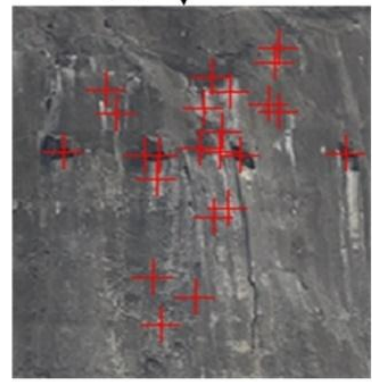

(d)

Figure 13. Synthetic images matching. (a,b) shows the matching results of synthetic images from the first and second station for test field in Figure 10b; (c,d) illustrates part of the results.

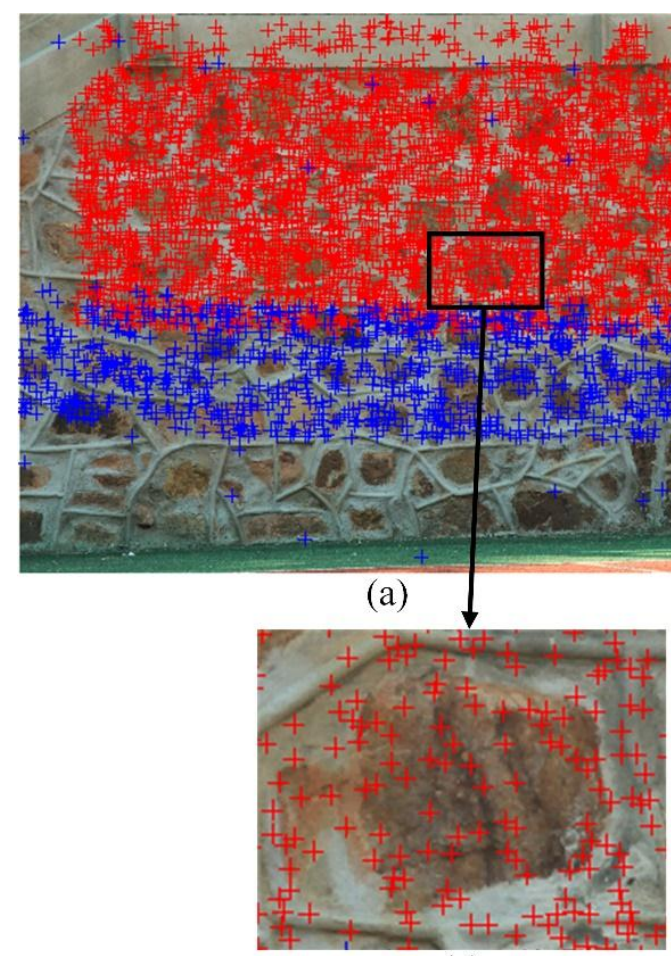

(c)

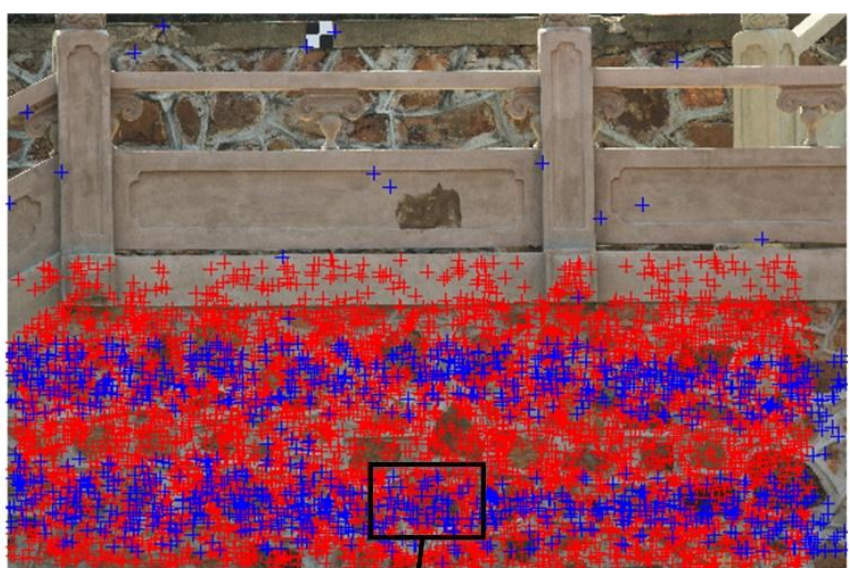

(b)

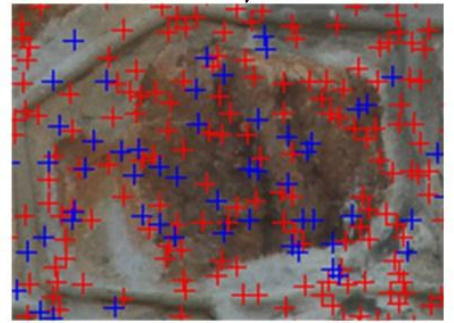

(d)

Figure 14. Original images matching. (a,b) shows the matching results of images from the adjacent stations as measuring the first field showed in Figure 10a; (c,d) illustrates part of the results. 


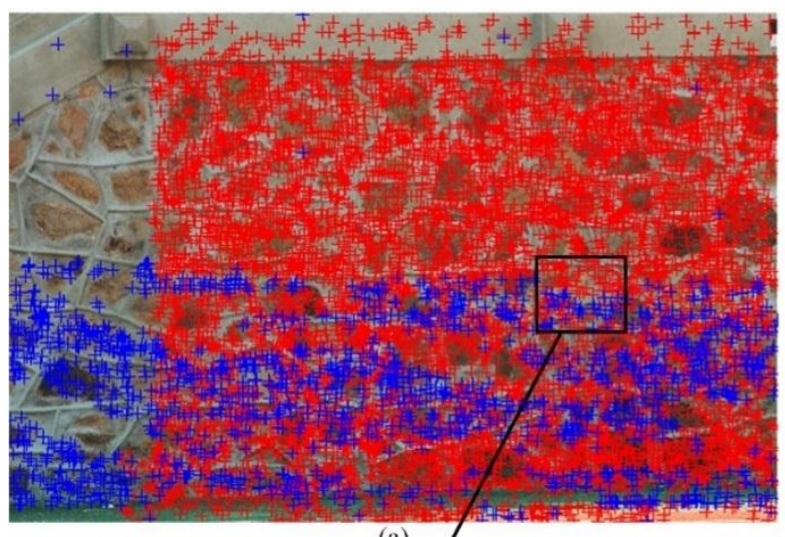

(a)

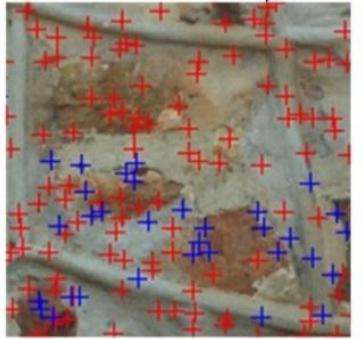

(c)

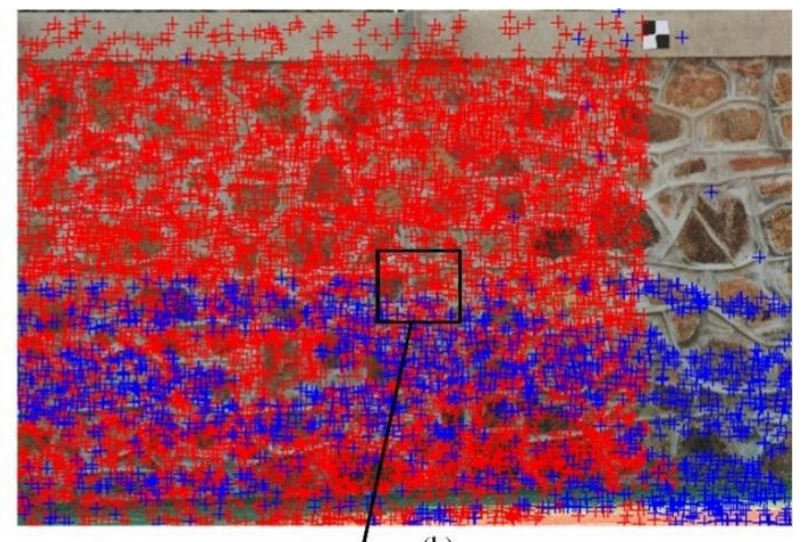

(b)

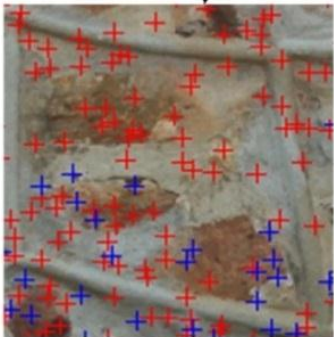

(d)

Figure 15. Original images matching. (a,b) shows the matching results of adjacent images in the same row of one image matrix as measuring the first field showed in Figure 10a; (c,d) illustrates part of the results.

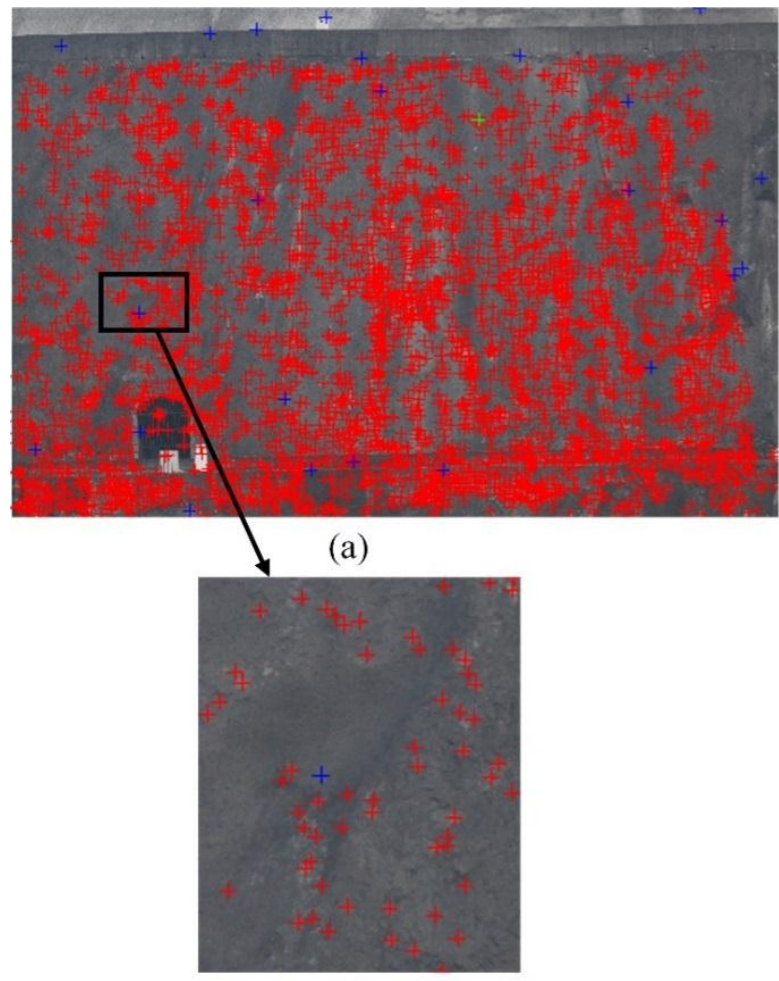

(c)

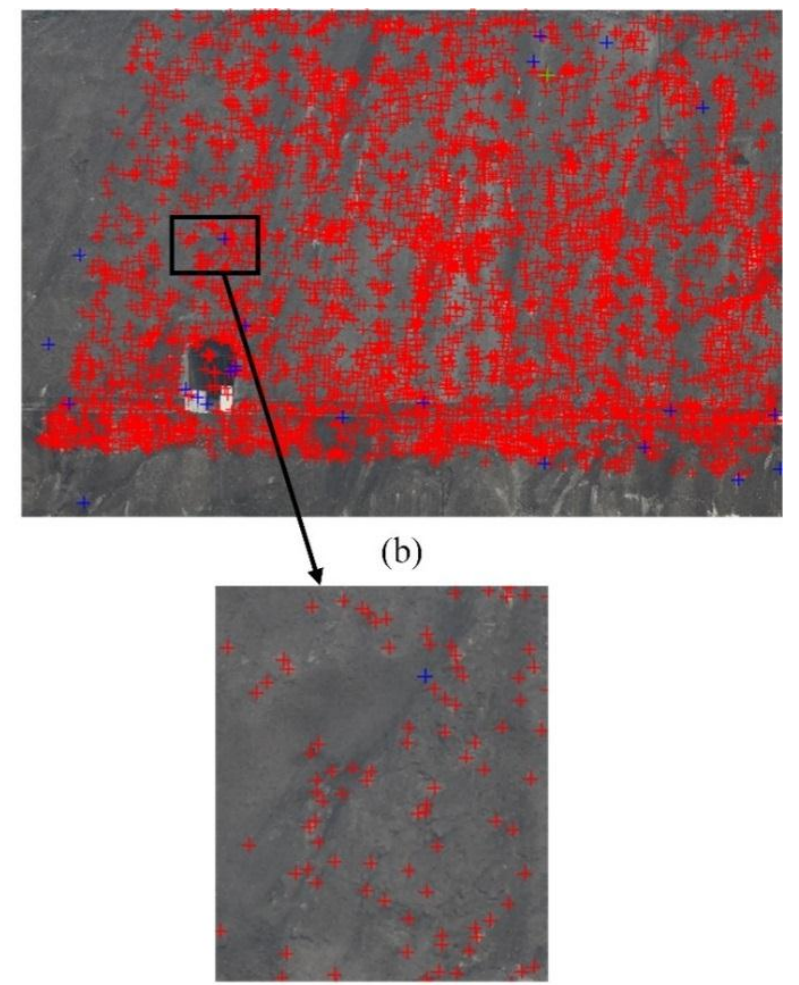

(d)

Figure 16. Results of stereo images matching. (a,b) shows the matching results of images from the adjacent stations as measuring the second field showed in Figure 10b; $(\mathbf{c}, \mathbf{d})$ illustrates part of the results. 


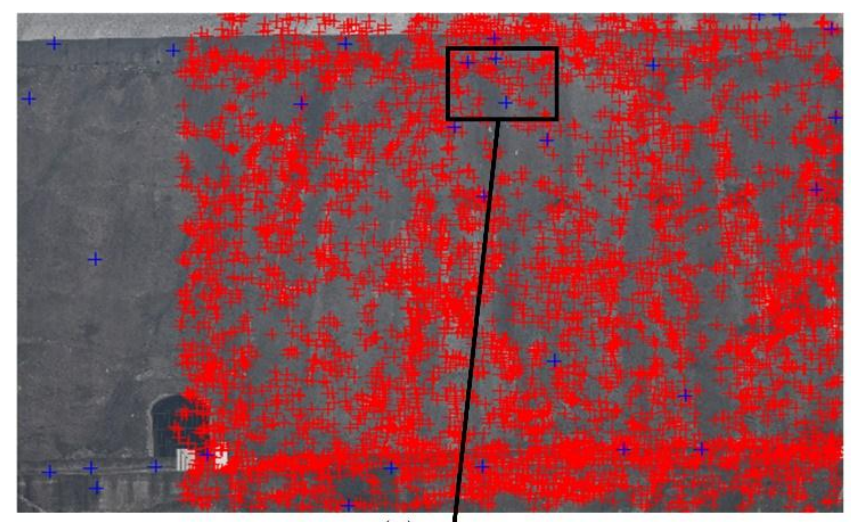

(a)

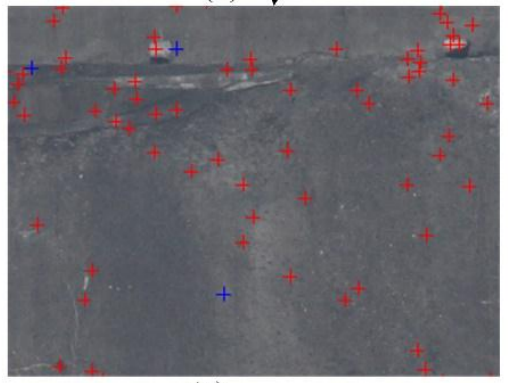

(c)

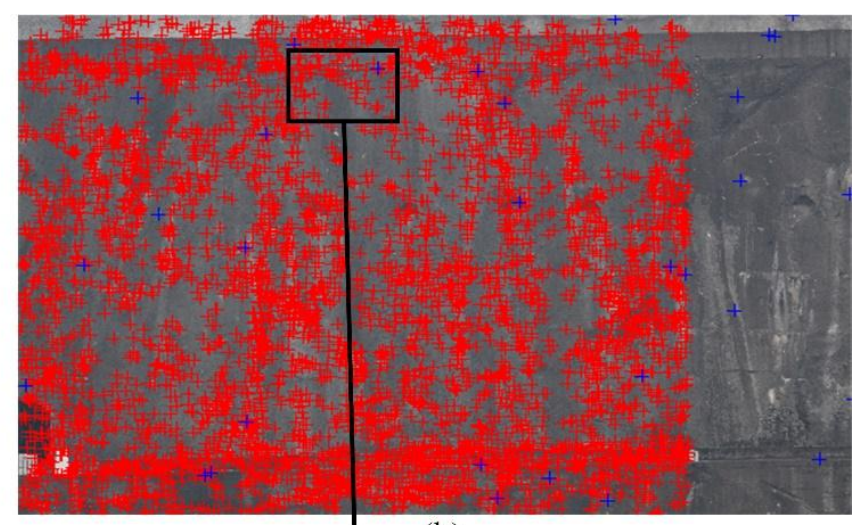

(b)

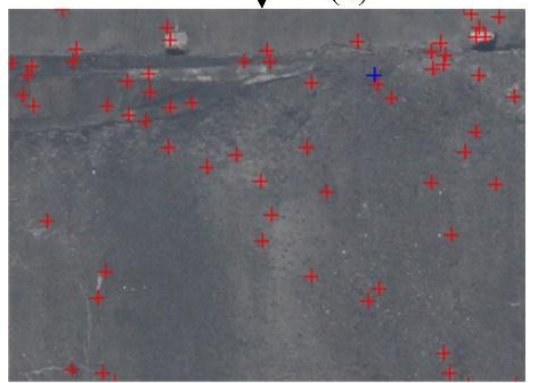

(d)

Figure 17. Results of stereo images matching. $(\mathbf{a}, \mathbf{b})$ shows the matching results of adjacent images in the same row of one image matrix as measuring the second field showed in Figure 10b; (c,d) illustrates part of the results.

\subsection{Results of Point Clouds}

The free network of original images is generated after the correspondences of original images are tied together. The generated point cloud is a mess, because exterior parameters of images are not computed accurately and interior parameters of the camera have not been calibrated at the moment. After selfcalibration bundle adjustment with control points, the point cloud will become regular and present the sketchy model of the target. Figures 18 and 19 show the difference of the point cloud of two test fields before and after self-calibration bundle adjustment with control points.

\subsection{Coordinate Residuals of Triangulation}

We conduct several groups of comparative experiments to evaluate the effects of different factors, such as photo distance, overlapping rate between original images in image matrices, given number of least stations $(M)$ from which any interest point on the target are acquired, set intersection angle $(\theta)$, and focal length, on the measurement accuracy in scanning photogrammetry. In the first test field of the flag platform, we analyze the effects of the four factors by using a camera with a $300 \mathrm{~mm}$ lens. In the second test field, the high slope at permanent lock of Three Gorges Project, two lenses with 300 and $600 \mathrm{~mm}$ lenses are used in evaluating the effects of focal length. 


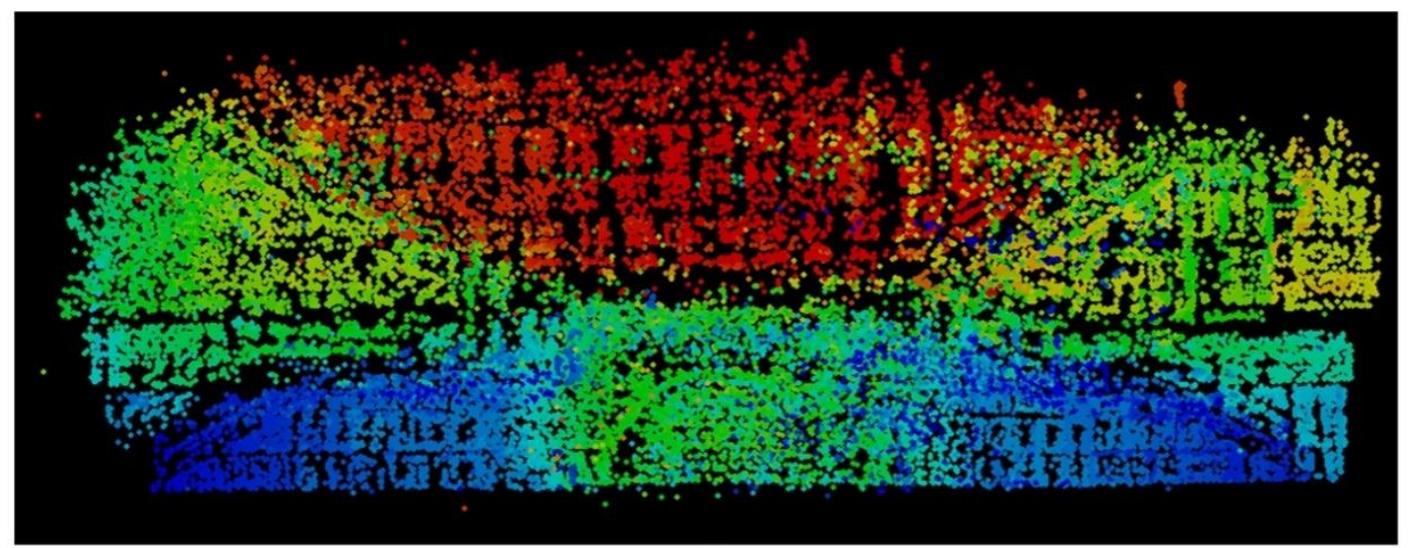

(a)

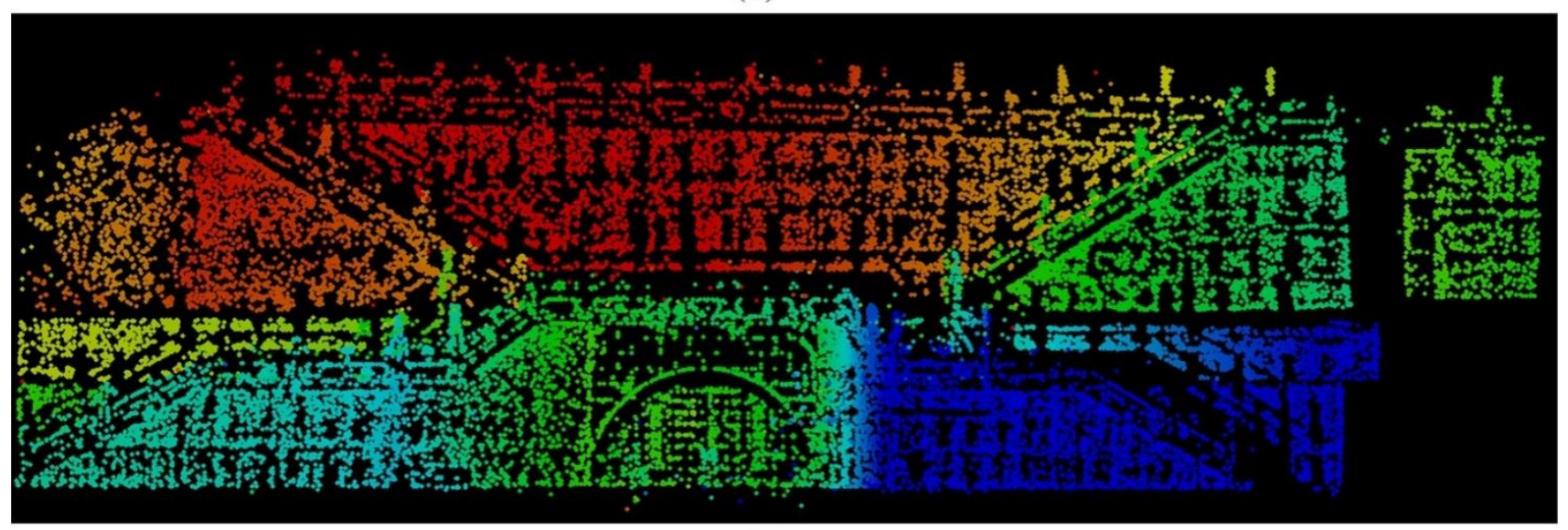

(b)

Figure 18. Point clouds. (a,b) shows the point clouds before and after self-calibration bundle adjustment with control points in the experiment of the first field showed in Figure 10a.

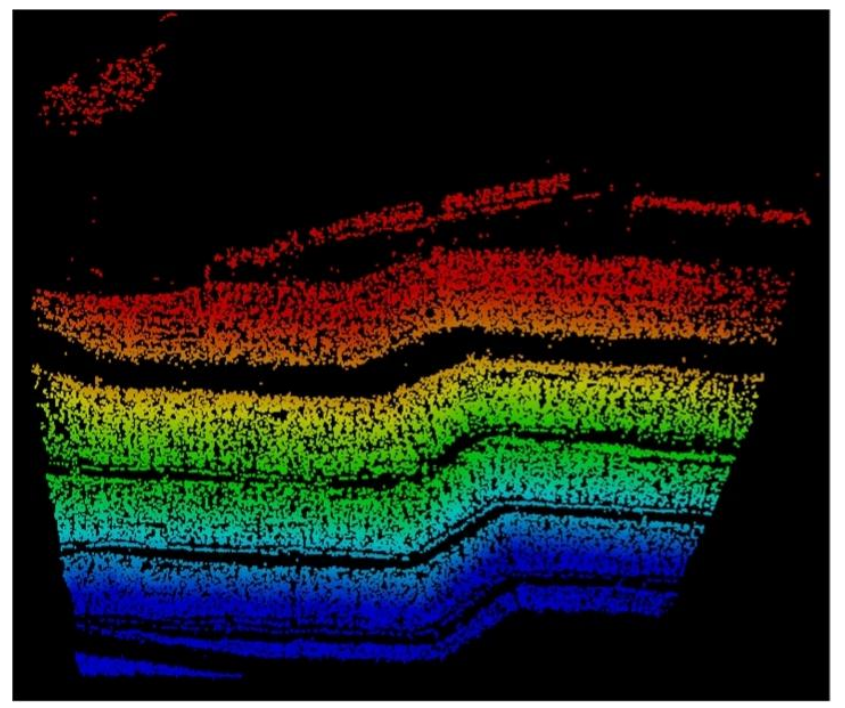

(a)

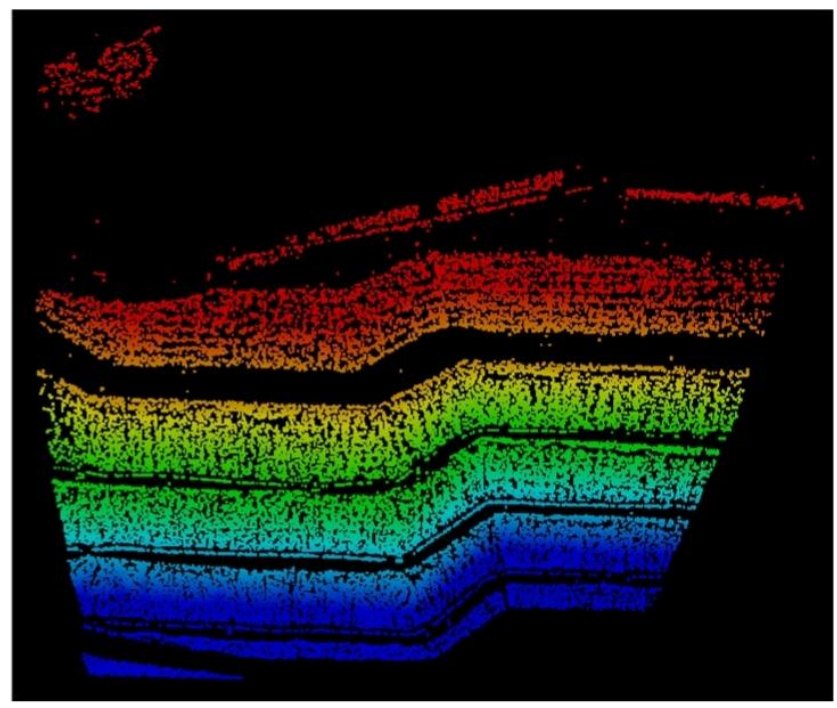

(b)

Figure 19. Point clouds. $(\mathbf{a}, \mathbf{b})$ shows the point clouds before and after bundle adjustment in the experiment of the second field showed in Figure 10b. 


\subsubsection{Comparison of Different Photo Distances}

To evaluate the measurement accuracy from different photo distances when using scanning photogrammetry, the images of the first test field are obtained from 40, 80, and $150 \mathrm{~m}$, and the ground resolutions are $0.9,1.7$, and $3.2 \mathrm{~mm}$, respectively. Experiments are correspondingly named as cases I, II, and III. The horizontal and vertical overlaps between original images in image matrices in the three cases are set the same, $80 \%$ and $60 \%$, respectively. We adjust the baseline of the stations to ensure that the station numbers of the three experiments are equal; that is, the intersection angles of the three experiments are different.

Table 1 displays the details of this experiment, and Figure 20 shows the error vectors. To compare the influence of photo distances in scanning photogrammetry, we choose 12 points as control points from 26 points distributed evenly in the test area, and the rest are check points. Owing to the angle existing between the average plane of the measured target and the XY plane in the chosen object coordinates, the distribution of control and check points on the right side of the picture is denser than that on the left side of pictures. Moreover, as shown in Table 1, the mean intersection angle of tie points is smaller than the set least intersection angle. The reason is that many of the tie points only appear in two adjacent stations.

Table 1. Parameters and coordinate residuals of experiments for first test field at different photo distances.

\begin{tabular}{|c|c|c|c|c|c|c|}
\hline \multicolumn{2}{|c|}{ Cases } & $\mathbf{I}$ & II & II & III & III \\
\hline \multicolumn{2}{|c|}{ Photo distance (m) } & 40 & 80 & 80 & 150 & 150 \\
\hline \multicolumn{2}{|c|}{ Focal length (mm) } & & & 300 & & \\
\hline \multicolumn{2}{|c|}{ Ground resolution $(\mathrm{mm})$} & 0.9 & 1.7 & 1.7 & 3.2 & 3.2 \\
\hline \multicolumn{2}{|c|}{ Total station number $N$} & & & 5 & & \\
\hline \multicolumn{2}{|c|}{ Give least intersection angle $\theta\left({ }^{\circ}\right)$} & 26 & 35 & 35 & 35 & 35 \\
\hline \multicolumn{2}{|c|}{ Baseline (m) } & 8.8 & 14 & 14 & 26.3 & 26.3 \\
\hline \multicolumn{2}{|c|}{ Image amount } & 858 & 171 & 171 & 41 & 41 \\
\hline \multicolumn{2}{|c|}{ RMSE of image point residuals (pixel) } & $1 / 2$ & $1 / 2$ & $1 / 2$ & $1 / 2$ & $1 / 2$ \\
\hline \multicolumn{2}{|c|}{ Mean intersection angle of tie points $\left({ }^{\circ}\right)$} & 23.7 & 18.5 & 18.5 & 18.1 & 18.1 \\
\hline \multicolumn{2}{|c|}{ Maximum intersection angle of tie points $\left(^{\circ}\right)$} & 43.2 & 37.6 & 36.4 & 35.8 & 35.6 \\
\hline \multicolumn{2}{|c|}{ Minimum intersection angle of tie points $\left({ }^{\circ}\right)$} & 4.2 & 4.4 & 4.8 & 6.3 & 6.5 \\
\hline \multicolumn{2}{|c|}{ Number of control points } & 12 & 12 & 10 & 12 & 8 \\
\hline \multirow{5}{*}{$\begin{array}{c}\text { Accuracy } \\
(\mathrm{mm})\end{array}$} & $\mathrm{X}$ & 1.2 & 1.8 & 1.6 & 1.5 & 1.5 \\
\hline & $\mathrm{Y}$ & 1.0 & 1.3 & 0.7 & 1.2 & 0.4 \\
\hline & $\mathrm{Z}$ & 1.1 & 2.2 & 2.7 & 2.5 & 2.9 \\
\hline & $\mathrm{XY}$ & 1.5 & 2.2 & 1.7 & 1.9 & 1.5 \\
\hline & $\mathrm{XYZ}$ & 1.9 & 3.1 & 3.2 & 3.1 & 3.3 \\
\hline \multicolumn{2}{|c|}{ Number of check points } & 14 & 14 & 16 & 14 & 18 \\
\hline \multirow{5}{*}{$\begin{array}{c}\text { Accuracy } \\
\text { (mm) }\end{array}$} & $\mathrm{X}$ & 2.0 & 2.1 & 2.4 & 2.5 & 2.5 \\
\hline & Y & 0.8 & 1.8 & 1.9 & 1.6 & 1.7 \\
\hline & Z & 1.0 & 2.5 & 2.5 & 2.8 & 2.7 \\
\hline & $X Y$ & 2.1 & 2.8 & 3.0 & 2.9 & 3.0 \\
\hline & $\mathrm{XYZ}$ & 2.3 & 3.7 & 3.9 & 4.0 & 4.1 \\
\hline
\end{tabular}




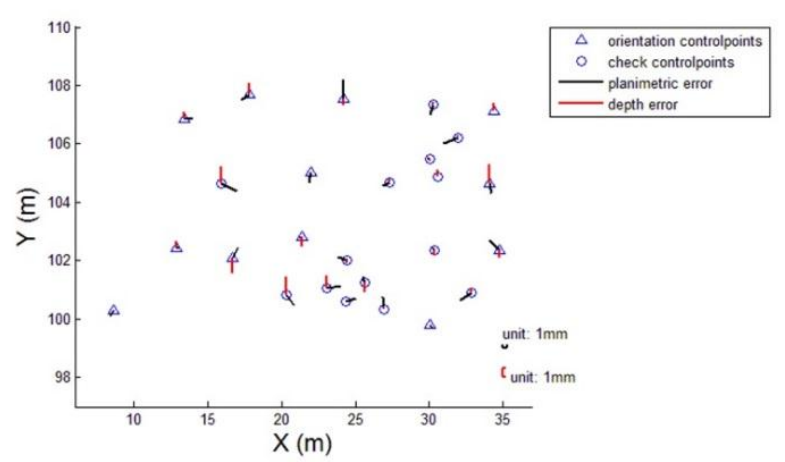

(a)

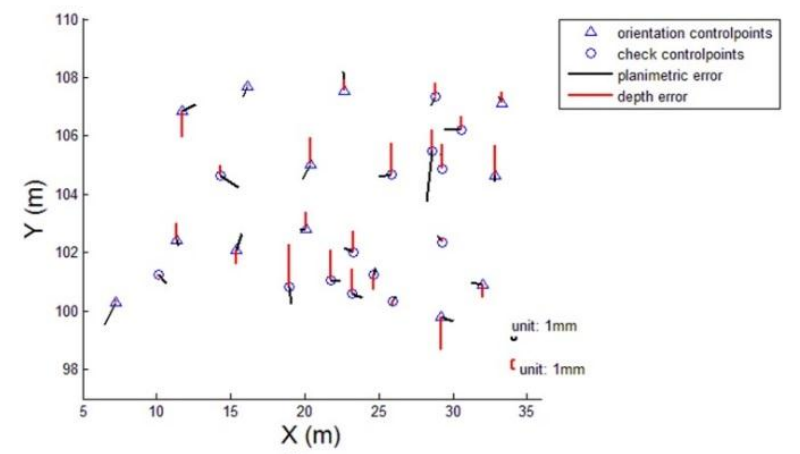

(b)

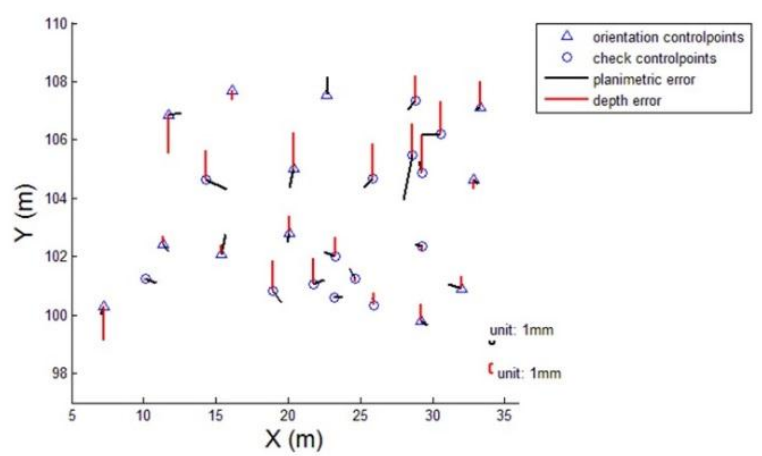

(d)

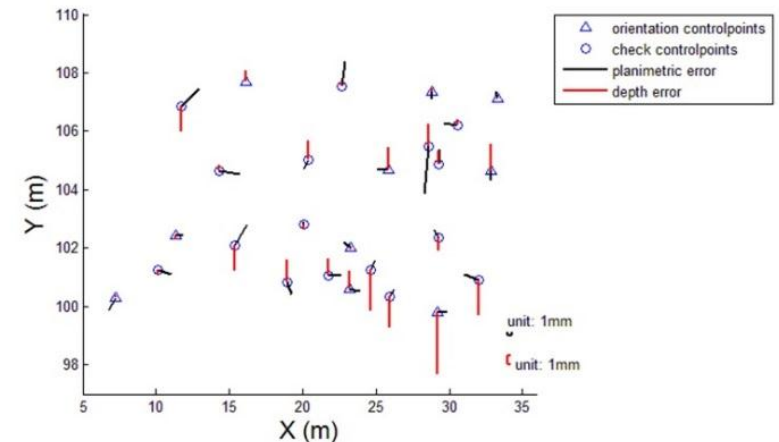

(c)

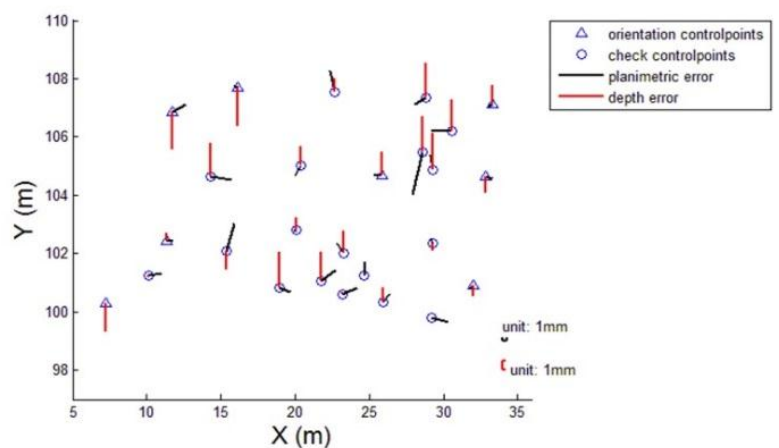

(e)

Figure 20. Error vectors of control points and check points. (a) is the error vectors of points measured at a distance of $40 \mathrm{~m}$; (b) and (c) denote error vectors of points measured at a photo distance of $80 \mathrm{~m}$. (b) is the result with 12 control points, and (c) is 10 control points; (d) and (e) show error vectors at a photo distance of $150 \mathrm{~m}$, and they are the results with 12 control points and eight control points, respectively.

As shown by the results in Table 1, scanning photogrammetry can achieve high accuracy even when measurement is performed from different photo distances. If ground sampling distance increases (which occurs if the photo distance also increases), then the pixel becomes larger when projected on the object, and less detail can be captured. As ground sampling distance increases, fine details become visible, which improves measurement accuracy. In this experiment, the accuracy of the control points measured by total station and the ground sampling distance are in the same magnitude, and the accuracy of the control points measured by total station is even larger than that of the ground sampling distance, thus causing the measurement accuracy to not be proportional to the photo distance. At the same time, the accuracy measured by the total station in the X-direction is the lowest. Therefore, in case I, the final 
measurement accuracy in the X-direction is lower than that in other directions because of its dependence on the accuracy measured by the total station. To further analyze the necessary amount of control points, we decrease the number of control points to 10 and 8 in cases II and III, respectively. The measurement results in Table 1 reveal that necessary control points decrease when the photo distance increases, which is related to the relief displacement of the target on images.

\subsubsection{Comparison of Different Overlaps between Images in Image Matrices}

In data acquisition, the number of original images that should be taken at one station is determined by the overlaps between images in image matrices. However, more images will relate to additional time spent on data acquisition and processing. Therefore, we prefer to minimize overlaps without affecting measurement accuracy. To analyze the influence of the horizontal overlapping rate in the image matrix on measurement accuracy, data with different horizontal overlaps $(80 \%, 60 \%$, and $30 \%)$ are obtained in the flag platform at a photo distance of $40 \mathrm{~m}$. The detailed parameters and coordinate residuals of control and check points are shown in Table 2. Figure 21 displays the error vectors. The experiment results reveal that horizontal overlaps in the image matrix affect measurement accuracy; that is, accuracy is lowered with the reduction of horizontal overlap. However, the influence is insignificant. We can deduce that the vertical overlap follows a similar way. Therefore, we can conclude that when the required precision is met, the horizontal and vertical overlaps can be decreased to improve the efficiency of data acquisition and processing. Meanwhile, experience dictates that the overlapping rate cannot be less than $30 \%$ in both directions to guarantee successive data processing.

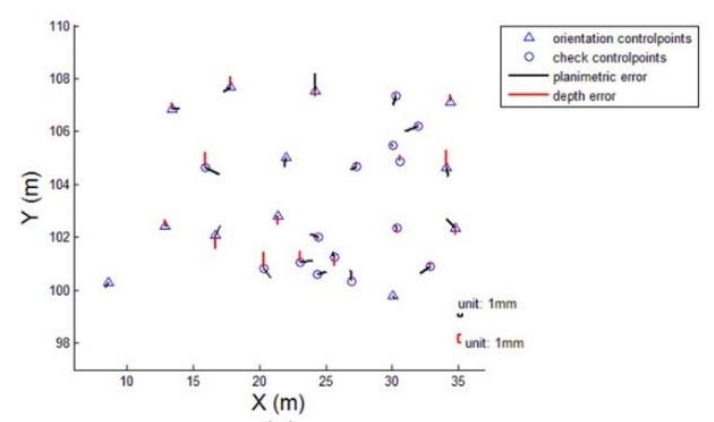

(a)

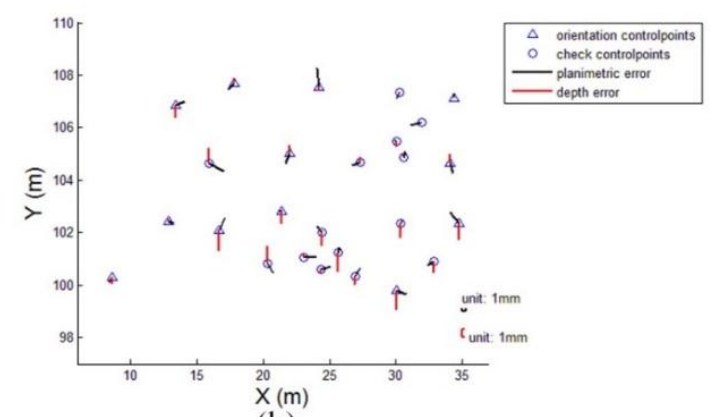

(b)

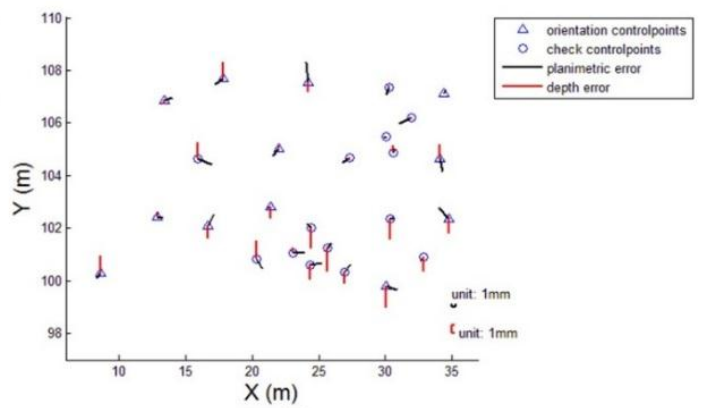

(c)

Figure 21. Error vectors of control points and check points. (a), (b), and (c) denote error vectors of points with horizontal overlap of $80 \%, 60 \%$, and $30 \%$, respectively. The coordinate residuals of check points in the experiments with image horizontal overlap $80 \%$, $60 \%$, and $30 \%$, respectively. 
Table 2. Parameters and coordinate residuals of experiments for first test field at different horizontal overlaps of image matrix.

\begin{tabular}{|c|c|c|c|c|}
\hline \multicolumn{2}{|c|}{ Cases } & $\mathbf{I}$ & II & III \\
\hline \multicolumn{2}{|c|}{ Photo distance (m) } & 40 & 40 & 40 \\
\hline \multicolumn{2}{|c|}{ overlap in horizontal direction } & $80 \%$ & $60 \%$ & $30 \%$ \\
\hline \multicolumn{2}{|c|}{ overlap in vertical direction } & $60 \%$ & $60 \%$ & $60 \%$ \\
\hline \multicolumn{2}{|c|}{ Focal length (mm) } & \multicolumn{3}{|c|}{300} \\
\hline \multicolumn{2}{|c|}{ Ground resolution $(\mathrm{mm})$} & \multicolumn{3}{|c|}{0.9} \\
\hline \multicolumn{2}{|c|}{ Total station number $N$} & \multicolumn{3}{|c|}{5} \\
\hline \multicolumn{2}{|c|}{ Give least intersection angle $\theta\left(^{\circ}\right)$} & \multicolumn{3}{|c|}{26} \\
\hline \multicolumn{2}{|c|}{ Baseline (m) } & \multicolumn{3}{|c|}{8.8} \\
\hline & & 858 & 444 & 232 \\
\hline RMSE of $i$ & iduals (pixel) & $1 / 2$ & $1 / 2$ & $1 / 2$ \\
\hline Mean inter & f tie points $\left(^{\circ}\right)$ & 23.7 & 23.4 & 22.5 \\
\hline Maximum int & of tie points $\left(^{\circ}\right)$ & 43.2 & 43.2 & 43.0 \\
\hline Minimum int & of tie points $\left({ }^{\circ}\right)$ & 4.2 & 5.6 & 2.4 \\
\hline Nur & points & 12 & 12 & 12 \\
\hline \multirow{5}{*}{$\begin{array}{c}\text { Accuracy } \\
(\mathrm{mm})\end{array}$} & $\mathrm{X}$ & 1.2 & 1.5 & 1.6 \\
\hline & $\mathrm{Y}$ & 1.0 & 1.1 & 1.2 \\
\hline & $\mathrm{Z}$ & 1.1 & 1.5 & 1.6 \\
\hline & $X Y$ & 1.5 & 1.9 & 2.0 \\
\hline & $\mathrm{XYZ}$ & 1.9 & 2.4 & 2.6 \\
\hline \multicolumn{2}{|c|}{ Number of check points } & 14 & 14 & 14 \\
\hline \multirow{5}{*}{$\begin{array}{c}\text { Accuracy } \\
(\mathrm{mm})\end{array}$} & $\mathrm{X}$ & 2.0 & 2.0 & 1.9 \\
\hline & $\mathrm{Y}$ & 0.8 & 0.7 & 0.7 \\
\hline & $\mathrm{Z}$ & 1.0 & 1.4 & 1.8 \\
\hline & $\mathrm{XY}$ & 2.1 & 2.1 & 2.1 \\
\hline & $\mathrm{XYZ}$ & 2.3 & 2.5 & 2.7 \\
\hline
\end{tabular}

\subsubsection{Comparison of $\mathrm{M}$}

According to the station distribution in our scanning photogrammetry, the baseline between stations decreases as the value of the given number of least stations from which any interest point on the target would be photographed $(M)$ increases. A shorter baseline is known to be conducive to image matching; however, the number of images in the project will be increased, which would require more time for data acquisition and processing. To analyze the influence of $M$, we conduct two experiments that measure the flag platform from three and five stations each at a photo distance of $80 \mathrm{~m}$. The parameters and coordinate residuals of control points and check points are listed in Table 3. Figure 22 shows the error vectors of these points. In this experiment, the relief is small from this photo distance, suggesting that $M$ rarely influences measurement accuracy. However, if the relief is large, $M$ should be increased. We recommend that the value of $M$ should be no less than 3 . 
Table 3. Parameters and coordinate residuals of experiments for first test field at different values of $M$.

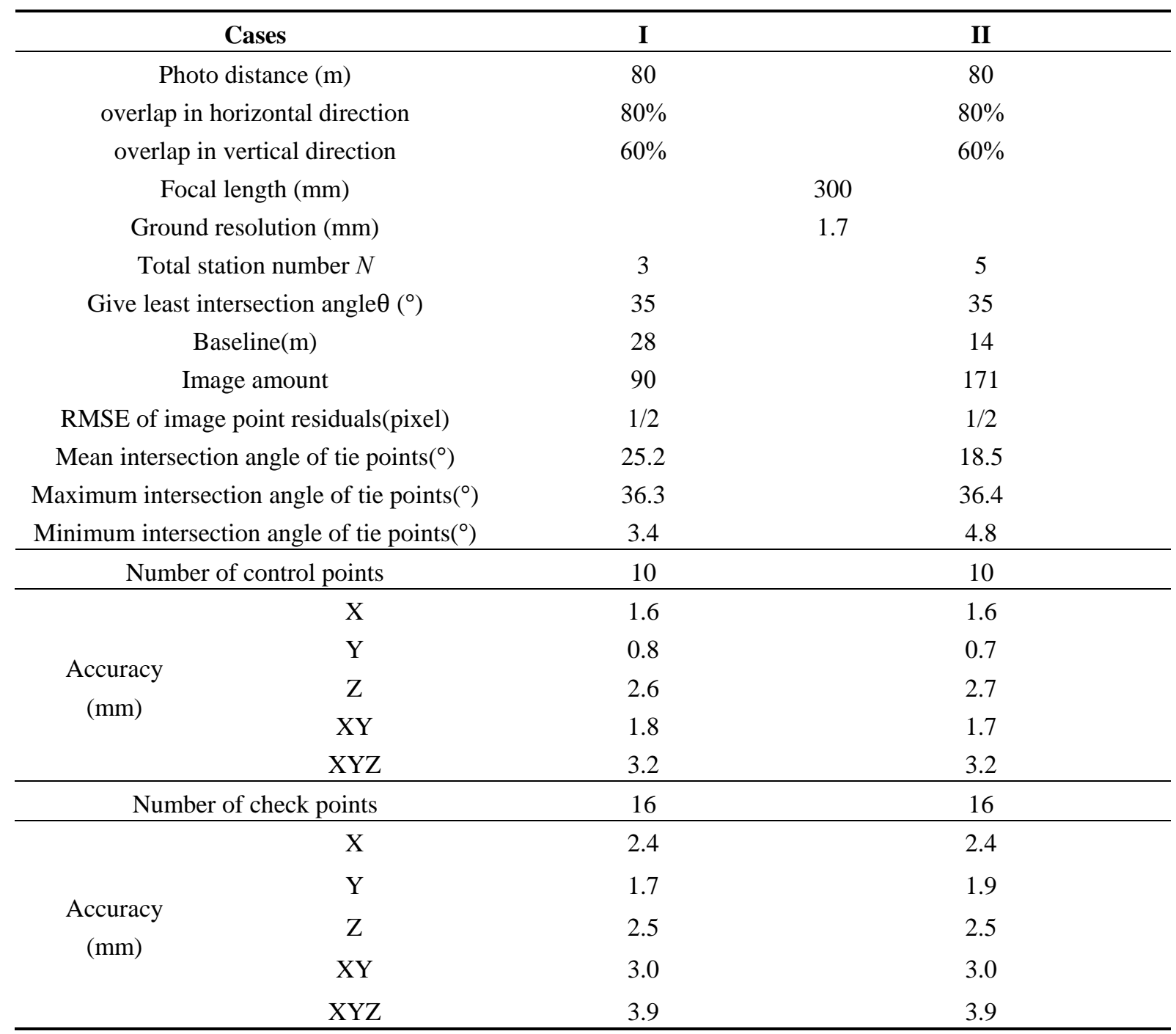

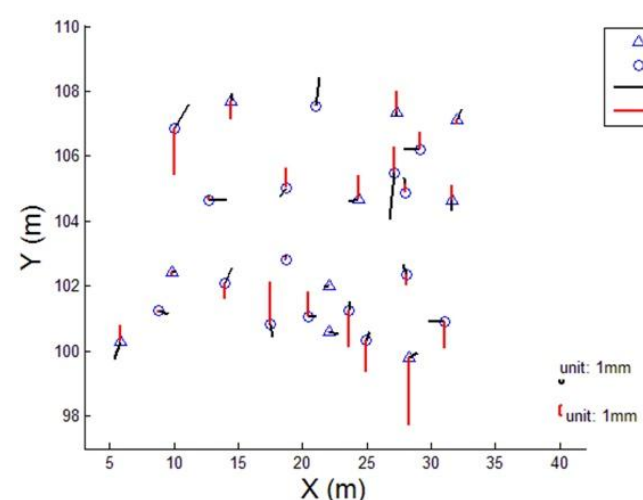

(a)

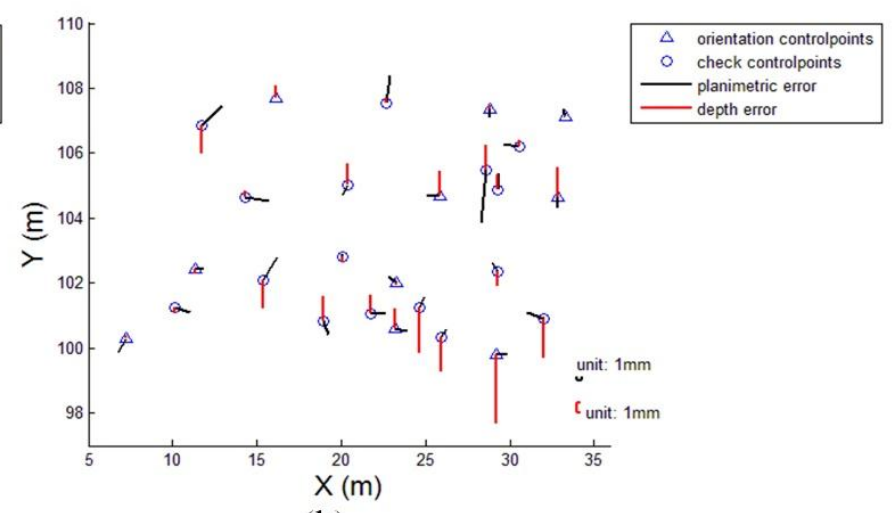

(b)

Figure 22. Error vectors of control points and check points. (a) and (b) denote error vectors of points in the experiments of obtaining images from three and five stations Coordinates residuals of check points in the experiments with $M$ as 3 and 5, respectively. 


\subsubsection{Comparison of Set Intersection Angle}

In this part, experiments with different intersection angles are conducted. The intersection angle is set to $26^{\circ}$ and $35^{\circ}$ in the two tests at a photo distance of $40 \mathrm{~m}$. According to the abovementioned rules of station distribution, the amounts of total stations are 3 and 5, respectively. Table 4 lists the detailed parameters of the experiments and the coordinate residuals. Figure 23 shows the error vectors. The results unexpectedly demonstrate that a larger intersection angle corresponds to a lower measurement accuracy. We speculate that the main cause of this situation is increase in the photo distortion when the intersection angle is enlarged. Therefore, a large intersection angle is not always better than a smaller intersection angle and should be determined according to the relief displacement of the measuring target.

Table 4. Parameters and coordinate residuals of experiments for first test field at different intersection angles.

\begin{tabular}{|c|c|c|c|}
\hline \multicolumn{2}{|c|}{ Cases } & $\mathbf{I}$ & II \\
\hline \multicolumn{2}{|c|}{ Photo distance (m) } & 40 & 40 \\
\hline \multicolumn{2}{|c|}{ overlap in horizontal direction } & $80 \%$ & $80 \%$ \\
\hline \multicolumn{2}{|c|}{ overlap in vertical direction } & $60 \%$ & $60 \%$ \\
\hline \multicolumn{2}{|c|}{ Focal length $(\mathrm{mm})$} & \multicolumn{2}{|c|}{300} \\
\hline \multicolumn{2}{|c|}{ Ground resolution $(\mathrm{mm})$} & \multicolumn{2}{|c|}{0.9} \\
\hline \multicolumn{2}{|c|}{ Total station number $N$} & 5 & 3 \\
\hline \multicolumn{2}{|c|}{ Give least intersection angle $\theta\left({ }^{\circ}\right)$} & 26 & 35 \\
\hline \multicolumn{2}{|c|}{ Baseline (m) } & 8.8 & 17.6 \\
\hline \multicolumn{2}{|c|}{ Image amount } & 858 & 738 \\
\hline \multicolumn{2}{|c|}{ RMSE of image point residuals (pixel) } & $1 / 2$ & $1 / 2$ \\
\hline \multicolumn{2}{|c|}{ Mean intersection angle of tie points $\left({ }^{\circ}\right)$} & 23.7 & 29.9 \\
\hline \multicolumn{2}{|c|}{ Maximum intersection angle of tie points $\left({ }^{\circ}\right)$} & 43.2 & 44.8 \\
\hline \multicolumn{2}{|c|}{ Minimum intersection angle of tie points $\left(^{\circ}\right)$} & 4.2 & 6.1 \\
\hline \multicolumn{2}{|c|}{ Number of control points } & 12 & 12 \\
\hline \multirow{5}{*}{$\begin{array}{l}\text { Accuracy } \\
(\mathrm{mm})\end{array}$} & $\mathrm{X}$ & 1.1 & 1.3 \\
\hline & Y & 1.0 & 1.1 \\
\hline & $\mathrm{Z}$ & 1.2 & 1.4 \\
\hline & $\mathrm{XY}$ & 1.4 & 1.7 \\
\hline & $\mathrm{XYZ}$ & 1.9 & 2.2 \\
\hline \multicolumn{2}{|c|}{ Number of check points } & 14 & 16 \\
\hline \multirow{5}{*}{$\begin{array}{c}\text { Accuracy } \\
(\mathrm{mm})\end{array}$} & $\mathrm{X}$ & 2.0 & 1.8 \\
\hline & $\mathrm{Y}$ & 0.8 & 0.8 \\
\hline & $\mathrm{Z}$ & 1.0 & 1.9 \\
\hline & $\mathrm{XY}$ & 2.1 & 2.0 \\
\hline & $\mathrm{XYZ}$ & 2.3 & 2.7 \\
\hline
\end{tabular}




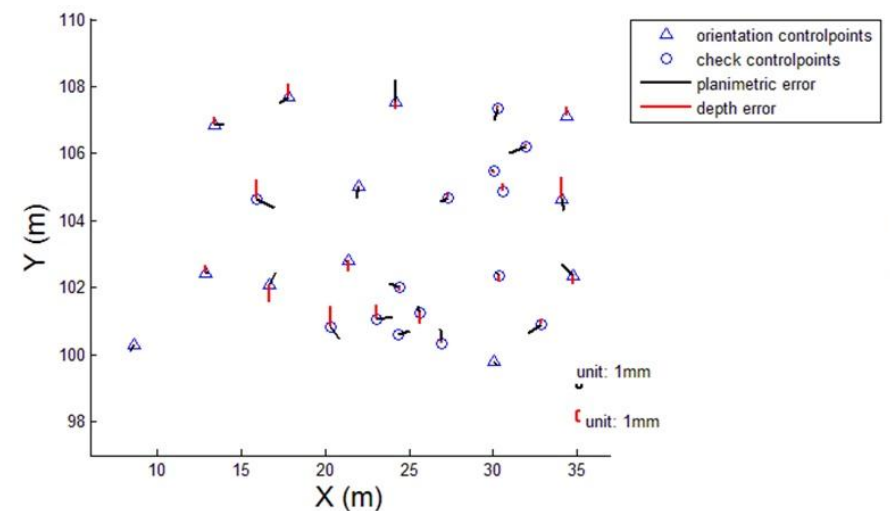

(a)

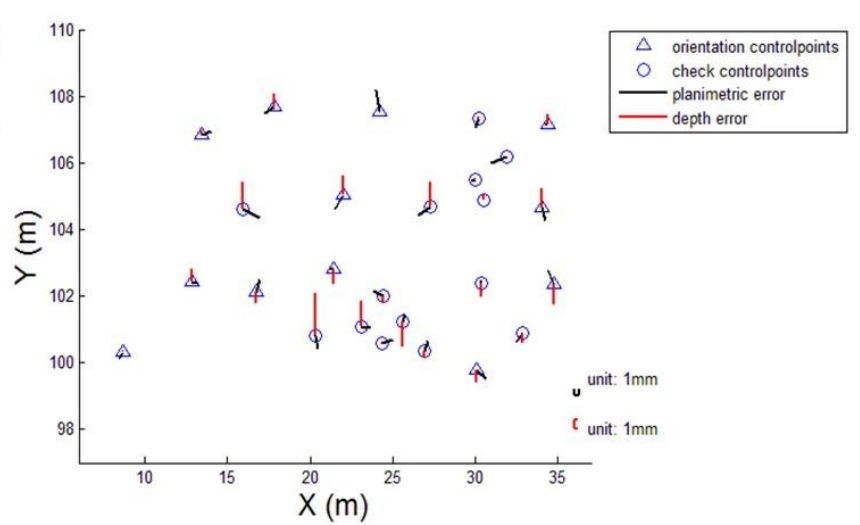

(b)

Figure 23. Error vectors of control points and check points. (a) and (b) demonstrate error vectors of points in the experiments with designed intersection angle as $26^{\circ}$ and $35^{\circ}$.

\subsubsection{Comparison of Different Focuses}

Ground sampling distance can be improved by increasing the focal length of the camera, which can be beneficial to measurement accuracy. In this part, two lenses with focus lenses of $300 \mathrm{~mm}$ and $600 \mathrm{~mm}$ are used for measuring the high slope in the second field (Figure 10b). The tests are named Cases I and II, respectively. Table 5 shows the detailed parameters of the experiments and the coordinate residuals, and Figure 24 illustrates the error vectors. The scene of the field is complex and thus can only be surveyed at a long distance of $250 \mathrm{~m}$. A total of 14 control points are selected for orientation. Table 5 shows that our scanning photogrammetry can achieve high precision when a large target from a long distance is measured. This efficient measurement in Case II reveals that our method is valid and can apply telephoto lens to achieve high precision with a few control points. However, the residuals in case II is expected to improve much more than that in case I because the ground sampling distance in case II is improved to half of that in case I. However, the accuracy was not improved substantially because the accuracy was limited by the accuracy of the control points measured by the total station. Another reason is the systematic errors that cannot been corrected completely because the distortion in images obtained with telephoto lenses is complicated. Therefore, finding ways to solve this problem is the main concern of our future works.

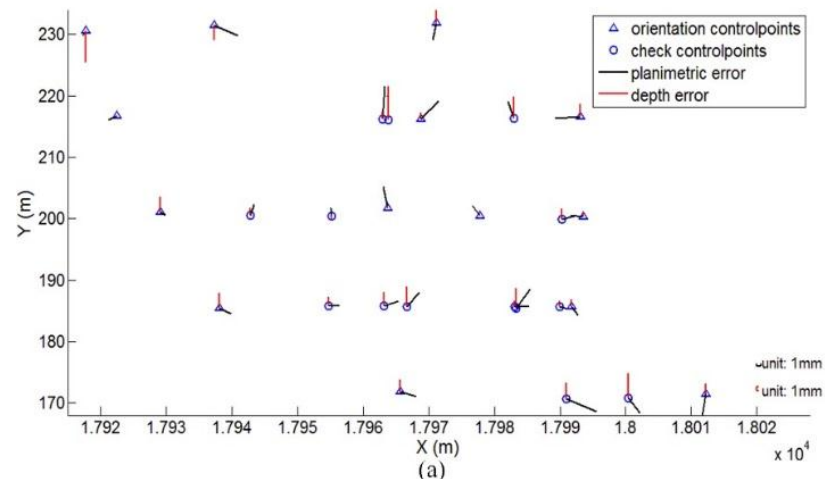

(a)

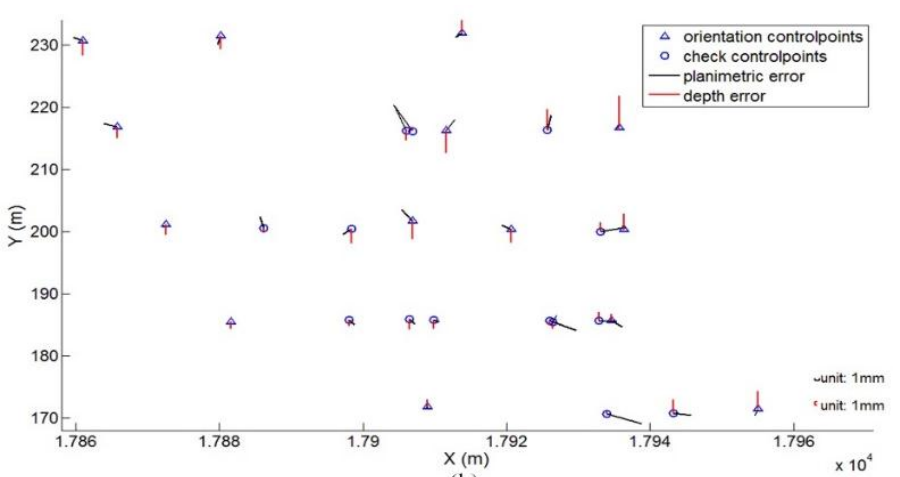

(b)

Figure 24. Error vectors of control points and check points. (a) and (b) demonstrate error vectors of points in the experiments with 300 and $600 \mathrm{~mm}$ focal lens. 
Table 5. Parameters and coordinate residuals of experiments for second test field at different focuses.

\begin{tabular}{|c|c|c|c|}
\hline \multicolumn{2}{|c|}{ Cases } & I & II \\
\hline \multicolumn{2}{|c|}{ Photo distance (m) } & 250 & 250 \\
\hline \multicolumn{2}{|c|}{ overlap in horizontal direction } & $80 \%$ & $60 \%$ \\
\hline \multicolumn{2}{|c|}{ overlap in vertical direction } & $60 \%$ & $40 \%$ \\
\hline \multicolumn{2}{|c|}{ Focal length (mm) } & 300 & 600 \\
\hline \multicolumn{2}{|c|}{ Ground resolution (mm) } & 5.3 & 2.7 \\
\hline \multicolumn{2}{|c|}{ Total station number $N$} & 3 & 3 \\
\hline \multicolumn{2}{|c|}{ Give least intersection angle $\theta\left({ }^{\circ}\right)$} & 30 & 30 \\
\hline \multicolumn{2}{|c|}{ Baselines (m) } & 55 & 55 \\
\hline \multicolumn{2}{|c|}{ Image amount } & 601 & 982 \\
\hline \multicolumn{2}{|c|}{ RMS of image point residuals (pixel) } & $2 / 5$ & $1 / 2$ \\
\hline \multicolumn{2}{|c|}{ Mean intersection angle of tie points $\left(^{\circ}\right)$} & 22.2 & 23.4 \\
\hline \multicolumn{2}{|c|}{ Maximum intersection angle of tie points $\left(^{\circ}\right)$} & 33.1 & 29.4 \\
\hline \multicolumn{2}{|c|}{ Minimum intersection angle of tie points $\left(^{\circ}\right)$} & 6.1 & 6.0 \\
\hline \multicolumn{2}{|c|}{ Number of control points } & 14 & 14 \\
\hline \multirow{5}{*}{$\begin{array}{l}\text { Accuracy } \\
(\mathrm{mm})\end{array}$} & $\mathrm{X}$ & 3.3 & 1.7 \\
\hline & $\mathrm{Y}$ & 3.3 & 1.6 \\
\hline & $\mathrm{Z}$ & 3.9 & 4.4 \\
\hline & $X Y$ & 4.6 & 2.3 \\
\hline & $\mathrm{XYZ}$ & 6.0 & 5.0 \\
\hline \multicolumn{2}{|c|}{ Number of check points } & 14 & 14 \\
\hline \multirow{5}{*}{$\begin{array}{c}\text { Accuracy } \\
(\mathrm{mm})\end{array}$} & $\mathrm{X}$ & 3.2 & 2.7 \\
\hline & $\mathrm{Y}$ & 4.0 & 3.2 \\
\hline & $\mathrm{Z}$ & 4.4 & 3.9 \\
\hline & XY & 5.1 & 4.2 \\
\hline & $\mathrm{XYZ}$ & 6.8 & 5.7 \\
\hline
\end{tabular}

\section{Discussion}

This paper uses scanning photography to solve the problem of data acquisition and organization caused by the limited FOV of digital cameras when measuring large targets with long focal lens. When cameras with long focal lens are used to measure large targets, the ground coverage is small, resulting in the need to take lots of images. If all these images are taken manually, then only experienced photographers can accomplish the work well. Otherwise, coverage gaps will occur, especially when applying multi-view photography to obtain images to maintain measurement precision. Scanning photography combined with the isometric station distribution proposed in this paper is designed to obtain images regularly, as in data acquisition in aerial photogrammetry. Also, it is a kind of photogrammetry method using multi-view images. After operators input the required parameters, the photo scanner automatically obtains images, thus making it easy to acquire images without coverage gap and to organize images for data processing. Moreover, the photo scanner can record the rotation angle of each image when obtaining images, which can be used in data processing as auxiliary information. A modified triangulation is conducted according to the traits of the data acquired by scanning photography that the 
overlaps among images from the same station are known, improving the efficiency and stability of data processing. The modified triangulation can be considered as a kind of global structure from motion (SFM) with auxiliary information.

Successfully used in aerial and close-range photogrammetry, scanning photography is the approach of controlling the camera to rotate around one point to acquire images to enlarge the field-view-of one station. Vision Map A3 system is an example of scanning photography in aerial photogrammetry. The camera used in this system consists of dual CCD detectors with two $300 \mathrm{~mm}$ lenses. As the flight moves when obtaining images, the camera can only be controlled to rotate in a cross-track direction, which provides a field-of-view of $104^{\circ}$. In the along-track direction, the field-of-view is enlarged by using dual CCDs. Traditional triangulation with GPS information is utilized for data processing. After that, super large frames synthesized from all pairs of original images of one sweep are generated for stereo photogrammetric mapping [24]. In close-range, the platform for obtaining images is static and always controls only one camera rotating in both horizontal and vertical directions (the same as along-crack and cross-crack direction) to enlarge the field of view. While, several systems take images both with normal and long focal lenses in measurements; normal focal lens images are used to provide a general network around the object, whereas the long focal lens images are employed to ensure accuracy and reconstruct the 'sharp' details [30-33]. And, the relative angles between adjacent images from one station are always the same in these systems.

The differences of our scanning photogrammetry and the previous ones are listed as follows:

(1) Difference in scanning photography

To avoid the situation when images at the corners of the image matrix are covered by invalid areas (as in Figure 5), which results from central perspective when photographing, the relative rotation angle between adjacent images decreases as the rotation angle increases from the "zero position", instead of staying the same in the previous scanning photography. Considering our scanning photogrammetry is used for measuring targets outside, which always allow only one side of the object towards the camera, we recommend that the horizontal rotation angle should be in the range of $\left[-45^{\circ}, 45^{\circ}\right]$, and that the vertical rotation angle should be within $\left[-30^{\circ}, 30^{\circ}\right]$ to limit image distortion. So that, our simplified synthesis method of projecting the image matrix on a plane is enough to actually deliver the application.

(2) Difference in rotation platform

For easy data acquisition, the photo scanner is designed to obtain images automatically. The weight of the long focal lens, particularly telephoto, may be heavier than that of the camera body; hence, the center of rotation and the center of camera are designed in different locations to enable the photo scanner to be successfully used with telephoto, as shown in Figure 7. However, the offset values of the two centers are small compared with the photo distance when large engineering measurements are measured using this photo scanner. Thus, we ignore the offset values when image matrices are synthesized.

(3) Difference in data processing

According to the mechanism of our scanning photography and station distribution, the overlap between ground coverages of adjacent image matrices is known and large enough for relative orientation, whereas the overlap between original stereo images from adjacent stations are unknown and may be 
insufficient for relative orientation. Therefore, we modify the traditional triangulation by utilizing synthetic images to calculate the initial parameters for bundle adjustment. The offset in $\mathrm{X}, \mathrm{Y}, \mathrm{Z}$ coordinates from the center of the camera to the center of rotation is very small compared to the photo distance in most cases; here, we ignore the difference when image matrices are synthesized. Then, a simplified approach is employed for generating synthetic images, which do not need the surface model of the target. Although model errors exist in the synthetic image, they do not have much influence on the matching of synthetic images, especially when the photo distance is large. After synthetic images are generated, they are used to generate a free network, and the initial exterior orientation parameters of the original images are calculated accordingly. Original images are employed when bundle adjustment is performed because perspective centers of each image from the same station are actually different and multi-directional images will contribute to accuracy and robustness. The accurate exterior parameters of the original images different from parameters of the corresponding synthetic images can be obtained after bundle adjustment.

Two reasons that our scanning photogrammetry can effectively process images with long focal length.

(1) The modified triangulation ensures the initial parameters are stable, which benefits for the convergence of bundle adjustment.

(2) In consideration of the unstable intrinsic parameters of the non-metric cameras with long focal lens, especially telephoto, the on-line self-calibration bundler adjustment with control points is employed in our scanning photogrammetry. We hold that the intrinsic parameters are constant during the short time of data acquisition. Further, we fixed the focus of lens with a tape to avoid small changes caused by vibration when the photo scanner moves. During bundler adjustment, the weights of observations based on intersection angles are considered to ensure its convergence.

However, the narrow field of view of long focal length can impact adversely on the linear dependencies between the interior and exterior orientation parameters [45]. Although bundle adjustment convergences in the experiments, the results do not meet expectations. The precision can be improved with proper calibration model. In future works, we will research this aspect.

\section{Conclusions}

In this paper, scanning photogrammetry was employed to improve the efficiency of large object measurement in close range. The limited format of digital cameras prevents normal photography when large targets are measured with long focal lens, which leads to low efficiency because of the difficult data acquisition and organization when using oblique photography as a substitution. This method was derived to acquire images in order as in traditional aerial photogrammetry, which was easily conducted and could avoid coverage gaps. Therefore, we improved the scanning photography, provided a design of station distribution, and developed the corresponding data acquisition instrument to ensure image data were obtained in an orderly way. Then, considering the traits of data acquired in our method, a modified triangulation is performed by utilizing synthetic images from image matrices to generate the free network to avoid matching all original images with one another firstly; then, yielding a free network from original images according to the free network from synthetic images; finally, executing self-calibration bundle adjustment with control points to calculate the final results. This modified triangulation method highly 
improved the efficiency and stability of data processing when using long focal lens for measurements. Also, the results of experiments confirmed that our method could achieve high measurement precision with 300 and $600 \mathrm{~mm}$ lenses, when using a small number of control points.

However, the following problems remain to be solved in future works.

(1) To apply our method to more kinds of targets, such as tunnels, we consider projecting the image matrix on a cylinder or a sphere in future research.

(2) To enhance measurement accuracy, we will continue to work on dealing with the image distortion using telephoto lens.

(3) In the next step, we will work towards calibration of the rotation platform to give more precise initial parameters for bundle adjustment.

\section{Acknowledgments}

This work was supported in part by the National Natural Science Foundation of China under Grants 41201482, the Research Funds of the Key Laboratory of Mapping from Space, National Administration of Surveying, Mapping and Geoinformation with project number K201405, the Fundamental Research Funds for the Central Universities with project number 2014213020201, and the Geographic National Condition Monitoring Engineering Research Center of Sichuan Province with project number GC201514. The authors would like to thank the anonymous reviewers, members of the editorial team, He Huang and Jianan He from Wuhan University, China, for their comments and contributions, which have significantly improved this paper.

\section{Author Contributions}

Shan Huang designed and implemented the algorithm and performed the experiments, she also wrote the paper; Zuxun Zhang directed the algorithm and experiment design and proposed to do the scanning photogrammetry; Tao Ke directed the algorithm and experiment design; Min Tang and Xuan Xu designed and made the photo-scanner.

\section{Conflicts of Interest}

The authors declare no conflict of interest.

\section{References and Notes}

1. Kim, K.Y.; Kim, C.Y.; Lee, S.D.; Seo, Y.S.; Lee, C.I. Measurement of tunnel 3-D displacement using digital photogrammetry. J. Korean Soc. Eng. Geol. 2007, 17, 567-576.

2. Baldi, P.; Fabris, M.; Marsella, M.; Monticelli, R. Monitoring the morphological evolution of the Sciara del Fuoco during the 2002-2003 Stromboli eruption using multi-temporal photogrammetry. ISPRS J. Photogramm. 2005, 59, 199-211.

3. Nakai, T.; Ryu, M.; Miyauchi, H.; Miura, S.; Ohnishi, Y.; Nishiyama, S. Underground Space Use: Analysis of the Past and Lessons for the Future; Taylor \& Francis: Istanbul, Turkey, 2005; pp. 1203-1209. 
4. Previtali, M.; Barazzetti, L.; Scaioni, M.; Tian, Y. An automatic multi-image procedure for accurate 3D object reconstruction. In Proceedings of the 2011 IEEE 4th International Congress on Image and Signal Processing (CISP), Shanghai, China, 15-17 October 2011; Volume 3, pp. 1400-1404.

5. Balletti, C.; Guerra, F.; Tsioukas, V.; Vernier, P. Calibration of Action Cameras for Photogrammetric Purposes. Sensors 2014, 14, 17471-17490.

6. Flener, C.; Vaaja, M.; Jaakkola, A.; Krooks, A.; Kaartinen, H.; Kukko, A.; Kasvi, E.; Hyyppä, H.; Hyyppä, J.; Alho, P. Seamless Mapping of river channels at high resolution using mobile LiDAR and UAV-Photography. Remote Sens. 2013, 5, 6382-6407.

7. Chandler, J. Effective application of automated digital photogrammetry for geomorphological research. Earth Surf. Proc. Land 1999, 24, 51-63.

8. Fraser, C.S.; Shortis, M.R.; Ganci, G. Multi-sensor system self-calibration. Proc. SPIE 1995, 2598, doi:10.1117/12.220890.

9. Peipe, J.; Schneider, C.T. High-resolution still video camera for industrial photogrammetry. Photogramm. Rec. 1995, 15, 135-139.

10. Feng, W. The application of non-metric camera in close-range photogrammetry. Railway Investig. Surv. 1982, 4, 43-54.

11. Rieke-Zapp, D.H. A Digital Medium-Format Camera for Metric Applications. Photogramm. Rec. 2010, 25, 283-298.

12. Yakar, M. Using close range photogrammetry to measure the position of inaccessible geological features. Exp. Tech. 2011, 35, 54-59.

13. Yilmaz, H.M. Close range photogrammetry in volume computing. Exp. Tech. 2010, 34, 48-54.

14. Luhmann, T. Close range photogrammetry for industrial applications. ISPRS J. Photogramm. 2010, $65,558-569$.

15. Fraser, C.S.; Cronk, S. A hybrid measurement approach for close-range photogrammetry. ISPRS J. Photogramm. 2009, 64, 328-333.

16. Ke, T.; Zhang, Z.X.; Zhang, J.Q. Panning and multi-baseline digital close-range photogrammetry. Proc. SPIE 2007, 6788, doi:10.1117/12.749055.

17. Ordonez, C.; Martinez, J.; Arias, P.; Armesto, J. Measuring building facades with a low-cost close-range photogrammetry system. Automat. Constr. 2010, 19, 742-749.

18. Dall'Asta, E.; Thoeni, K.; Santise, M.; Forlani, G.; Giacomini, A.; Roncella, R. Network Design and Quality Checks in Automatic Orientation of Close-Range Photogrammetric Blocks. Sensors 2015, 15, 7985-8008.

19. Fraser, C.S.; Edmundson, K.L. Design and implementation of a computational processing system for off-line digital close-range photogrammetry. ISPRS J. Photogramm. 2000, 55, 94-104.

20. Fraser, C.S.; Woods, A.; Brizzi, D. Hyper redundancy for accuracy enhancement in automated close range photogrammetry. Photogramm. Rec. 2005, 20, 205-217.

21. Jiang, R.N.; Jauregui, D.V. Development of a digital close-range photogrammetric bridge deflection measurement system. Measurement 2010, 43, 1431-1438.

22. Geodetic Systems, Inc. Available online: http://www.geodetic.com/v-stars.aspx (accessed on 27 June 2015).

23. AICON 3D Systems. Available online: http://aicon3d.com/start.html (accessed on 27 June 2015). 
24. Pechatnikov, M.; Shor, E.; Raizman, Y. VisionMap A3 - super wide angle mapping system basic principles and workflow. In Proceeding of the 21th ISPRS Congress, Beijing, China, 3-11 July 2008; pp. 1735-1740.

25. Fangi, G.; Nardinocchi, C. Photogrammetric Processing of Spherical Panoramas. Photogram. Rec. 2013, 28, 293-311.

26. Takasu, M.; Sato, T.; Kojima, S; Hamada, K. Development of Surveying Robot. In Proceedings of the 13th International Association for Automation and Robotics in Construction, Tokyo, Japan, 11-13 June 1996; pp. 709-716.

27. Huang, Y.D. 3-D measuring systems based on theodolite-CCD cameras. Int. Arch. Photogramm. Remote Sens. Spat. Inf. Sci. 1993, 29, 541.

28. Ke, T.; Zhang, Z.; Zhang, J. Panning and multi-baseline digital close-range photogrammetry. Geomat. Inf. Sci. Wuhan Univ. 2009, 34, 44-47.

29. Ke, T. Panning and Multi-baseline Digital Close-range Photogrammetry. Ph.D. Thesis, Wuhan University, Wuhan, China, 2008.

30. Barazzetti, L.; Previtali, M.; Scaioni, M. 3D modeling from gnomonic projections. ISPRS Ann. Photogramm. Remote Sens. Spat. Inf. Sci. 2012, 1, 19-24.

31. Barazzetti, L.; Previtali, M.; Scaioni, M. Simultaneous registration of gnomonic projections and central perspectives. Photogramm. Rec. 2014, 29, 278-296.

32. Barazzetti, L.; Previtali, M.; Scaioni, M. Stitching and processing gnomonic projections for closerange photogrammetry. Photogramm. Eng. Remote Sens. 2013, 79, 573-582.

33. Fangi, G. Multiscale Multiresolution Spherical Photogrammetry with Long Focal Lenses for Architectural Surveys. Int. Arch. Photogramm. Remote Sens. Spat. Inf. Sci. 2010, 38, 1-6.

34. Amiri Parian, J.; Gruen, A. Sensor modeling, self-calibration and accuracy testing of panoramic cameras and laser scanners. ISPRS J. Photogramm. 2010, 65, 60-76.

35. Schneider, D.; Hans, M. A geometric model for linear-array-based terrestrial panoramic cameras. Photogram. Rec. 2006, 21, 198-210.

36. Mikhail, E.M.; Bethel, J.S.; McGlone, J.C. Introduction to Modern Photogrammetry; Wiley: New York, NY, USA, 2001; p. 479.

37. Maas, H.G.; Hampel, U. Photogrammetric techniques in civil engineering material testing and structure monitoring. Photogramm. Eng. Remote Sens. 2006, 72, 39-45.

38. Lowe, D.G. Distinctive Image Features from Scale-Invariant Keypoints. Int. J. Comput. Vis. 2004, $60,91-110$.

39. Hartley, R; Zisserman, A. Multiple View Geometry in Computer Vision, 2nd ed.; Cambridge University Press: New York, NY, USA, 2003; pp. 87-131.

40. Ma J.L.; Chan, J.C.; Canters, F. Fully Automatic Subpixel Image Registration of Multiangle CHRIS/Proba Data. IEEE Trans. Geosci. Remote Sens. 2010, 48, 2829-2839.

41. Nistér, D. An efficient solution to the five-point relative pose problem. IEEE Trans. Pattern Anal. Mach. Intell. 2004, 26, 756-770.

42. Stewenius, H.; Engels, C.; Nister, D. Recent developments on direct relative orientation. ISPRS J. Photogramm. 2006, 60, 284-294. 
43. Tee-Ann, T.; Liang-Chien, C.; Chien-Liang, L.; Yi-Chung, T.; Wan-Yu, W. DEM-Aided Block Adjustment for Satellite Images With Weak Convergence Geometry. IEEE Trans. Geosci. Remote Sens. 2010, 48, 1907-1918.

44. Slama, C.C.; Theurer C.; Hendrikson S.W. Manual of Photogrammetry, 4th ed.; American Society of Photogrammetry: Falls Church, VA, USA, 1980; p.1059.

45. Stamatopoulos, C.; Fraser, C.S. Calibration of long focal length cameras in close range photogrammetry. Photogramm. Rec. 2011, 26, 339-360.

(C) 2015 by the authors; licensee MDPI, Basel, Switzerland. This article is an open access article distributed under the terms and conditions of the Creative Commons Attribution license (http://creativecommons.org/licenses/by/4.0/). 